\title{
The genus Scaptodrosophila Duda (Diptera, Drosophilidae), part III: the riverata species group from China, with morphological and molecular evidence for five new species
}

\author{
Yong Lin', Hong-Wei Chen' \\ I Department of Entomology, South China Agricultural University, Tianhe, Guangzhou, 510642, China \\ Corresponding author: Hong-Wei Chen (hongweic@scau.edu.cn)
}

Academic editor: O. Lonsdale | Received 2 January 2020 | Accepted 7 April 2020 | Published 1 June 2020

http://zoobank.org/3772774D-6906-4106-8396-96CF4581D79A

Citation: Lin Y, Chen H-W (2020) The genus Scaptodrosophila Duda (Diptera, Drosophilidae), part III: the riverata species group from China, with morphological and molecular evidence for five new species. ZooKeys 937: 139-162. https://doi.org/10.3897/zookeys.937.49794

\begin{abstract}
A new species group, the riverata species group, is established within the genus Scaptodrosophila based on morphological and molecular evidence for five known and five new species from China: S. abdentata sp. nov., S. cederholmi (Okada, 1988), S. crocata (Bock, 1976), S. paraclubata (Sundaran \& Gupta, 1991), S. platyrhina sp. nov., S. puncticeps (Okada, 1956), S. riverata (Singh \& Gupta, 1977), S. serrateifoliacea sp. nov., S. sinuata sp. nov. and S. tanyrhina sp. nov. A key to this group is provided. Furthermore, 51 mtDNA COI sequences belonging to $S$. puncticeps, $S$. riverata and the five new species are used for verifying species boundaries defined by the morphological data.
\end{abstract}

\section{Keywords}

cryptic species, DNA barcoding, integrative taxonomy, molecular research, new species group

\section{Introduction}

A total of 12 species groups have been erected within the genus Scaptodrosophila (Duda 1923): albifrontata group (Wheeler and Takada 1966); aterrima group (Tsacas et al. 1988); barkeri group (Bock and Parsons 1978); brunnea group (Tsacas and Chassagnard 1976; Liu et al. 2017); brunneipennis group (Bock and Parsons 1978); bryani

Copyright Yong Lin, Hong-Wei Chen. This is an open access article distributed under the terms of the Creative Commons Attribution License (CC BY 4.0), which permits unrestricted use, distribution, and reproduction in any medium, provided the original author and source are credited. 
group (Throckmorton 1962); coracina group (Mather 1955; Liu and Chen 2018); inornata group (Parsons and Bock 1978); latifasciaeformis group (Burla 1954); ruffirons group (Papp et al. 1999); saba group (Burla 1954) and victoria group (Wheeler 1949). Together, these taxa include ca 300 species. Here we describe five new species from China that are morphologically similar to five known, yet unplaced, species: S. cederholmi (Okada, 1988) from Sri Lanka; S. crocata (Bock, 1976) from Australia; S. paraclubata (Sundaran \& Gupta, 1991) from India; S. puncticeps (Okada, 1956) from China, Kuril Islands, Korea, Japan; and S. riverata (Singh \& Gupta, 1977) from China, India, Myanmar. These ten species all have a yellowish-brown body; an arista with two dorsal and one ventral branch in addition to a terminal bifurcation; a large facial carina; and developed prescutellar setae. This morphological group is also supported by molecular data for the five new species and two of the five previously described taxa. The combined morphological and molecular evidence supports the establishment of a new species group, the riverata species group, based on five known and five new species from China.

DNA barcoding technology was employed to investigate the relationship of the riverata species group. Based on the results of the phylogenetic reconstruction, 51 barcode sequences of the $C O I$ (mitochondrial cytochrome $c$ oxidase subunit I) gene belonging to two known and five new species were used to evaluate these species boundaries.

\section{Materials and methods}

\section{Specimens}

The riverata group species were collected by net sweeping from tussocks and tree trunks. All the examined specimens were preserved in $75 \%$ ethanol.

\section{Species identification}

Total DNA was extracted from the abdominal tissue of samples after the dissection of the genitalia, using the TIANGEN ${ }^{\mathrm{TM}}$ DNA extraction kit following the recommended protocol. The COI fragments were amplified using the cycle protocol as in Zhao et al. (2009). The PCR sequencing primer pair was 5'-CGCCTAAACTTCAGCCACTT-3' (Wang et al. 2006) and 5'-TAAACTTCAGGGTGACCAAAAAATCA-3' (Folmer et al. 1994). All sequences generated in this study were supplied with BOLD Process ID and GenBank accession numbers (Table 1).

A total of 51 COI sequences of the riverata group were examined and aligned with MEGA 7.0 (Kumar et al. 2016). Intra- and interspecific genetic distances were calculated for species of the riverata group using the $p$-distance model (Nei and Kumar 2000). We also conducted character-based species barcoding where fixed sites of one species differed from those of the others; these were manually selected as diagnostic sites (i.e., "pure" diagnostics, Sarkar et al. 2002; Desalle et al. 2005). Three known species: S. melanogaster (GenBank accession number: KR070823), S. rhina (KR070845) and 
Table I. Specimens of brunnea species used for molecular study.

\begin{tabular}{|c|c|c|c|}
\hline & BOLD Process ID & $\begin{array}{c}\text { GenBank accession } \\
\text { number }\end{array}$ & Collection sites \\
\hline S. puncticeps -1 & BDORM010-14 & KJ841771 & Shennongjia, Hubei \\
\hline S. puncticeps -2 & BDORM011-14 & KJ841770 & Danba, Ganzizhou, Sichuan \\
\hline S. puncticeps -3 & BDORM012-14 & KJ841766 & Tianmushan, Linan, Zhejiang \\
\hline S. puncticeps -4 & BDORM013-14 & KJ841769 & Daozhen, Zunyi, Guizhou \\
\hline S. puncticeps -5 & BDORM014-14 & KJ841768 & Ailaoshan, Jingdong, Yunnan \\
\hline S. puncticeps -7 & BDORM004-14 & KJ841761 & Shennongjia, Hubei \\
\hline S. puncticeps -8 & BDORM005-14 & KJ841762 & Miyaluo, Abazhou, Sichuan \\
\hline S. riverata -1 & BDORM008-14 & KJ841773 & Banli, Chongzuo, Guangxi \\
\hline S. riverata -2 & BDORM009-14 & KJ841772 & Ailaoshan, Jingdong, Yunnan \\
\hline S. riverata -3 & SDLY001-19 & MK335597 & Likan, Ximeng, Yunnan \\
\hline S. riverata -4 & SDLY002-19 & MK335598 & Likan, Ximeng, Yunnan \\
\hline S. riverata -5 & SDLY003-19 & MK335599 & Likan, Ximeng, Yunnan \\
\hline S. riverata -6 & SDLY004-19 & MK335600 & Huanglianshan, Lvchun, Yunnan \\
\hline S. riverata -7 & SDLY005-19 & MK335601 & Huanglianshan, Lvchun, Yunnan \\
\hline S. abdentata sp. nov. -1 & BDORM019-14 & KJ841758 & Nanling, Shaoguan, Guangdong \\
\hline S. abdentata sp. nov. -2 & BDORM020-14 & KJ841757 & Muotuo, Lingzhi, Xizang \\
\hline S. abdentata sp. nov. -3 & BDORM021-14 & KJ841755 & Wangtianshu, Mengla, Yunnan \\
\hline S. abdentata sp. nov. -4 & SDLY006-19 & MK335586 & Muyiji, Ximeng, Yunnan \\
\hline S. abdentata sp. nov. -5 & BDORM023-14 & KJ841759 & Wuzhishan, Ledong, Hainan \\
\hline S. abdentata sp. nov. -6 & BDORM024-14 & KJ841756 & Muotuo, Lingzhi, Xizang \\
\hline S. abdentata sp. nov. -7 & SDLY007-19 & MK335587 & Muyiji, Ximeng, Yunnan \\
\hline S. abdentata sp. nov. -8 & SDLY008-19 & MK335588 & Mengdong, Cangyuan, Yunnan \\
\hline S. abdentata sp. nov. -9 & SDLY009-19 & MK335589 & Mengdong, Cangyuan, Yunnan \\
\hline S. platyrhina sp. nov. -1 & BDORM016-14 & KJ841765 & Menglun, Mengla, Yunnan \\
\hline S. platyrhina sp. nov. -2 & BDORM017-14 & KJ841764 & Menglun, Mengla, Yunnan \\
\hline S. platyrhina sp. nov. -3 & BDORM018-14 & KJ841763 & Jiangcheng, Simao, Yunnan \\
\hline S. platyrhina sp. nov. -4 & SDLY010-19 & MK335590 & Menglun, Mengla, Yunnan \\
\hline S. platyrhina sp. nov. -5 & SDLY011-19 & MK335591 & Guanleigang, Mengla, Yunnan \\
\hline S. platyrhinasp. nov. -6 & SDLY012-19 & MK335592 & Menglun, Mengla, Yunnan \\
\hline S. platyrhina sp. nov. -7 & SDLY013-19 & MK335593 & Menglun, Mengla, Yunnan \\
\hline S. platyrhina sp. nov. -8 & SDLY014-19 & MK335594 & Menglun, Mengla, Yunnan \\
\hline S. platyrhina sp. nov. -9 & SDLY015-19 & MK335595 & Menglun, Mengla, Yunnan \\
\hline S. platyrhina sp. nov. -10 & SDLY016-19 & MK335596 & Menglun, Mengla, Yunnan \\
\hline S. serrateifoliacea sp. nov. -1 & BDORM006-14 & KJ841775 & Hesong, Menghai, Yunnan \\
\hline S. serrateifoliacea sp. nov. -2 & BDORM007-14 & KJ841774 & Hesong, Menghai, Yunnan \\
\hline S. serrateifoliacea sp. nov. -3 & SDLY017-19 & MK335602 & Mengdong, Cangyuan, Yunnan \\
\hline S.sinuata sp.nov. -1 & SDLY024-19 & MK335603 & Dayangcha, Kuangdian, Liaoning \\
\hline S.sinuata sp.nov. -2 & SDLY025-19 & MK335604 & Dayangcha, Kuangdian, Liaoning \\
\hline S.sinuata sp.nov. -3 & SDLY026-19 & MK335605 & Laobiangou, Benxi, liaoning \\
\hline S.sinuata sp.nov. -4 & SDLY027-19 & MK335606 & Laobiangou, Benxi, liaoning \\
\hline S.sinuata sp.nov. -5 & SDLY028-19 & MK335607 & Laobiangou, Benxi, liaoning \\
\hline S.sinuata sp.nov. -6 & SDLY029-19 & MK335608 & Guojiapuzi, Kuangdian, Liaoning \\
\hline S.sinuata sp.nov. -7 & SDLY030-19 & MK335609 & Guojiapuzi, Kuangdian, Liaoning \\
\hline S.sinuata sp.nov. -8 & SDLY031-19 & MK335610 & Guojiapuzi, Kuangdian, Liaoning \\
\hline S. tanyrhina sp. nov. -1 & SDLY018-19 & MK335611 & Menglun, Mengla, Yunnan \\
\hline S. tanyrhina sp. nov. -2 & SDLY019-19 & MK335612 & Menglun, Mengla, Yunnan \\
\hline S. tanyrhina sp. nov. -3 & SDLY020-19 & MK335613 & Wangtianshu, Mengla, Yunnan \\
\hline S. tanyrhina sp. nov. -4 & SDLY021-19 & MK335614 & Wangtianshu, Mengla, Yunnan \\
\hline S. tanyrhina sp. nov. -5 & SDLY022-19 & MK335615 & Guanleigang, Mengla, Yunnan \\
\hline S. tanyrhina sp. nov. -6 & SDLY023-19 & MK335616 & Wangtianshu, Mengla, Yunnan \\
\hline
\end{tabular}


S. scutellimargo (KR070847), were used as outgroup taxa in the phylogenetic analyses. The alignment was subsequently employed to reconstruct a phylogenetic tree using the Neighbor-joining (NJ) method with $p$-distance model implemented in MEGA 7.0.26 (Kumar et al. 2016). Nodal support values (bootstrap percentages, BPs) were inferred by bootstrapping with 1000 replicates and other default settings.

\section{Description of species}

An Mshot Camera was used to photomicrograph all the examined species. All photographs, illustrations, and line drawings were processed with the software Adobe Photoshop 7.0 and Easy PaintTool SAI Ver.1.0.0. The morphological terminology follows McAlpine (1981) and the definitions of measurements, indices, and abbreviations follow Chen \& Toda (2001).

The type specimens were deposited in Department of Entomology, South China Agricultural University, Guangzhou, China (SCAU).

\section{Results}

The alignment of the 51 COI sequences spans 632 nucleotide sites, with 131 variable sites, 122 of which were parsimony informative. Intra- and interspecific $p$-distances were provided in Table 2 . The results show that the largest intraspecific $p$-distances within the riverata species group was 0.032 detected in $S$. puncticeps, followed by 0.016 in $S$. platyrhina sp. nov. while the minimum interspecific variation was 0.014 detected between $S$. abdentata sp. nov. and S. tanyrhina sp. nov.

The NJ (Fig. 1) tree shows that this new group is monophyletic with respect to the outgroups. Figure 2 shows nucleotides representing "pure" diagnostic sites for all species of the riverata group; at least one diagnostic site was recognized for each species. For example, site 21 is diagnostic for $S$. serrateifoliacea sp. nov. with a fixed status of $\mathrm{C}$ (Cytosine), rather than $\mathrm{T}$ (Thymidine) in the other species.

Table 2. Summary of intra- and interspecific genetic distances.

\begin{tabular}{lccc}
\hline \multicolumn{1}{c}{ Species } & N & intraspecific genetic distances & interspecific genetic distances \\
\cline { 2 - 4 } & & Min. /Max. /Mean \pm SD & Min. /Max. /Mean \pm SD \\
\hline S.puncticeps & 8 & $0.000 / 0.032 / 0.020 \pm 0.008$ & $0.071 / 0.127 / 0.107 \pm 0.017$ \\
S. riverata & 7 & $0.000 / 0.006 / 0.002 \pm 0.002$ & $0.049 / 0.138 / 0.084 \pm 0.032$ \\
S. abdentata & 9 & $0.000 / 0.006 / 0.003 \pm 0.002$ & $0.014 / 0.136 / 0.073 \pm 0.048$ \\
S. platyrhina & 10 & $0.000 / 0.016 / 0.007 \pm 0.004$ & $0.022 / 0.136 / 0.078 \pm 0.046$ \\
S. serrateifoliacea & 3 & $0.002 / 0.003 / 0.002 \pm 0.001$ & $0.070 / 0.138 / 0.116 \pm 0.026$ \\
S. sinuata & 8 & $0.000 / 0.009 / 0.005 \pm 0.003$ & $0.070 / 0.134 / 0.113 \pm 0.023$ \\
S. tanyrhina & 6 & $0.003 / 0.009 / 0.005 \pm 0.002$ & $0.014 / 0.134 / 0.070 \pm 0.048$ \\
\hline
\end{tabular}

$\mathrm{N}$, the numbers of $\mathrm{COI}$ sequences involved in distance calculation; Min., minimum; Max., maximum; SD, standard deviation; NA, no applicable. 


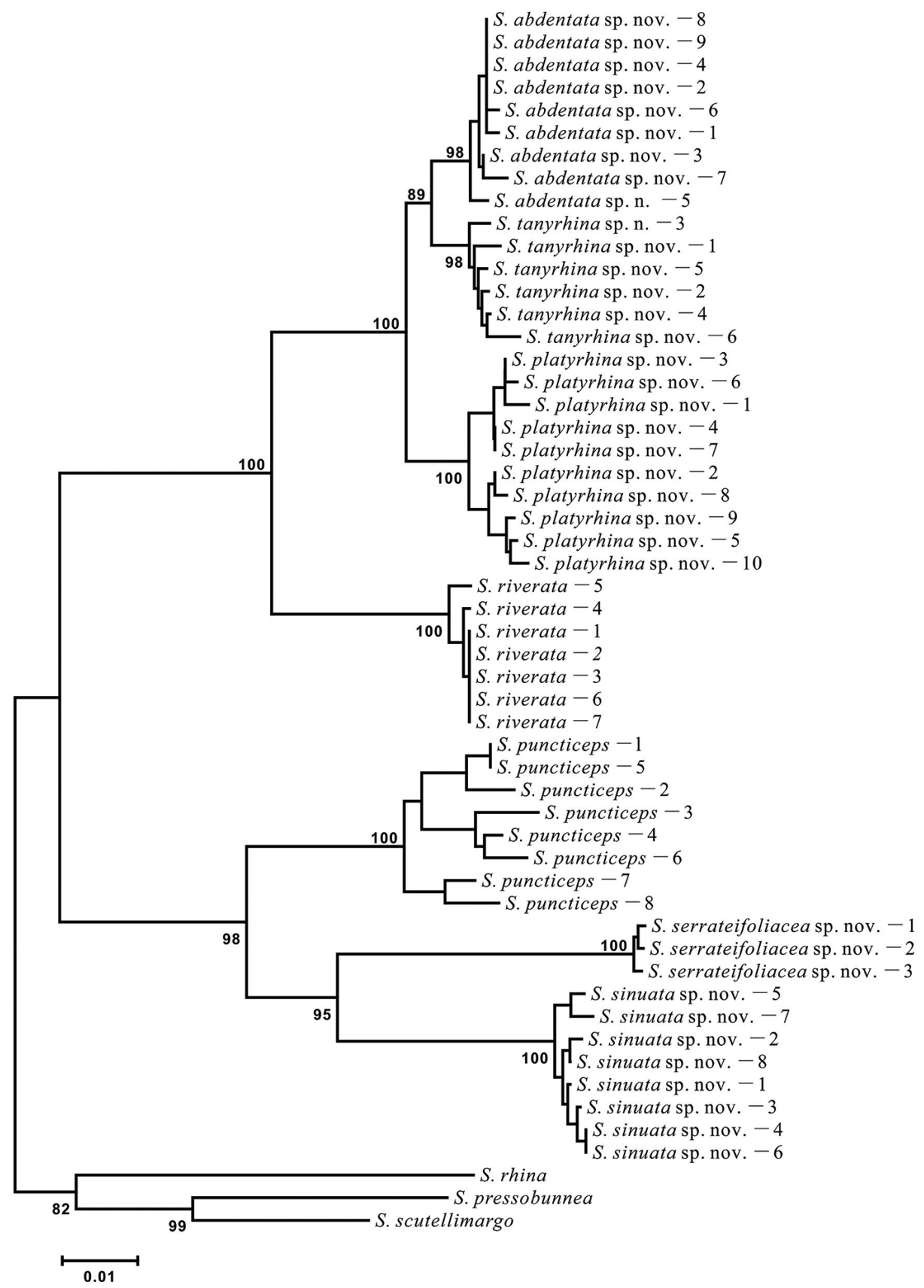

Figure I. Neighbor-joining (NJ) tree of the riverata group. The numbers around the nodes are bootstrap (BP) percentages. BP values lower than 50 are not shown. 
$\begin{array}{llllllllllllllllllllllllll}1 & 1 & 1 & 1 & 2 & 2 & 2 & 2 & 2 & 2 & 2 & 2 & 2 & 3 & 3 & 3 & 3 & 3 & 4 & 4 & 4 & 4 & 5 & 5 & 5 & 6\end{array}$

$\begin{array}{llllllllllllllllllllllllllllllll}2 & 3 & 4 & 8 & 9 & 0 & 0 & 2 & 7 & 1 & 2 & 3 & 3 & 7 & 8 & 8 & 8 & 9 & 0 & 3 & 5 & 8 & 8 & 2 & 4 & 5 & 8 & 2 & 3 & 5 & 1\end{array}$

S. puncticeps -1

S. puncticeps -2

$\begin{array}{lllllllllllllllllllllllllllllll}1 & 9 & 2 & 2 & 6 & 0 & 8 & 6 & 1 & 4 & 8 & 1 & 7 & 6 & 2 & 5 & 9 & 2 & 3 & 6 & 4 & 1 & 4 & 9 & 1 & 9 & 6 & 8 & 7 & 0 & 2\end{array}$

S. puncticeps -3

S. puncticeps -4

S. puncticeps -5

S. puncticeps -6

S. puncticeps -7

S. puncticeps -8

S. riverata -1

S. riverata -2

S. riverata -3

S. riverata -4

S. riverata -5

S. riverata -6

S. riverata -7

S. abdentata sp.nov. -1

S. abdentata sp.nov. -2

S. abdentata sp.nov. -3

S. abdentata sp.nov. -5

S. abdentata sp.nov. -4

S. abdentata sp.nov. -6

S. abdentata sp.nov. -7

S. abdentata sp.nov. -8

S. abdentata sp.nov. -9

S. platyrhina sp.nov. -1

S. platyrhina sp.nov. -2

S. platyrhina sp.nov. -3

S. platyrhina sp.nov. -4

S. platyrhina sp.nov. -5

S. platyrhina sp.nov. -6

S. platyrhina sp.nov. -7

S. platyrhina sp.nov. -8

S. platyrhina sp.nov. -9

S. platyrhina sp.nov. -10

S. serrateifoliacea sp.nov. -1

S. serrateifoliacea sp.nov. -2

S. serrateifoliacea sp.nov. -3

S. sinuata sp.nov. -1

S. sinuata sp.nov. -2

S. sinuata sp.nov. -3

S. sinuata sp.nov. -4

S. sinuata sp.nov. -5

S. sinuata sp.nov. -6

S. sinuata sp.nov. -7

S. sinuata sp.nov. -8

S. tanyrhina sp.nov. -1

S. tanyrhina sp.nov. -2

S. tanyrhina sp.nov. -3

S. tanyrhina sp.nov. -4

S. tanyrhina sp.nov. -5

S. tanyrhina sp.nov. -6

T A T T A T T A A C A T A C T A T T T A A A A A A T A T T A A

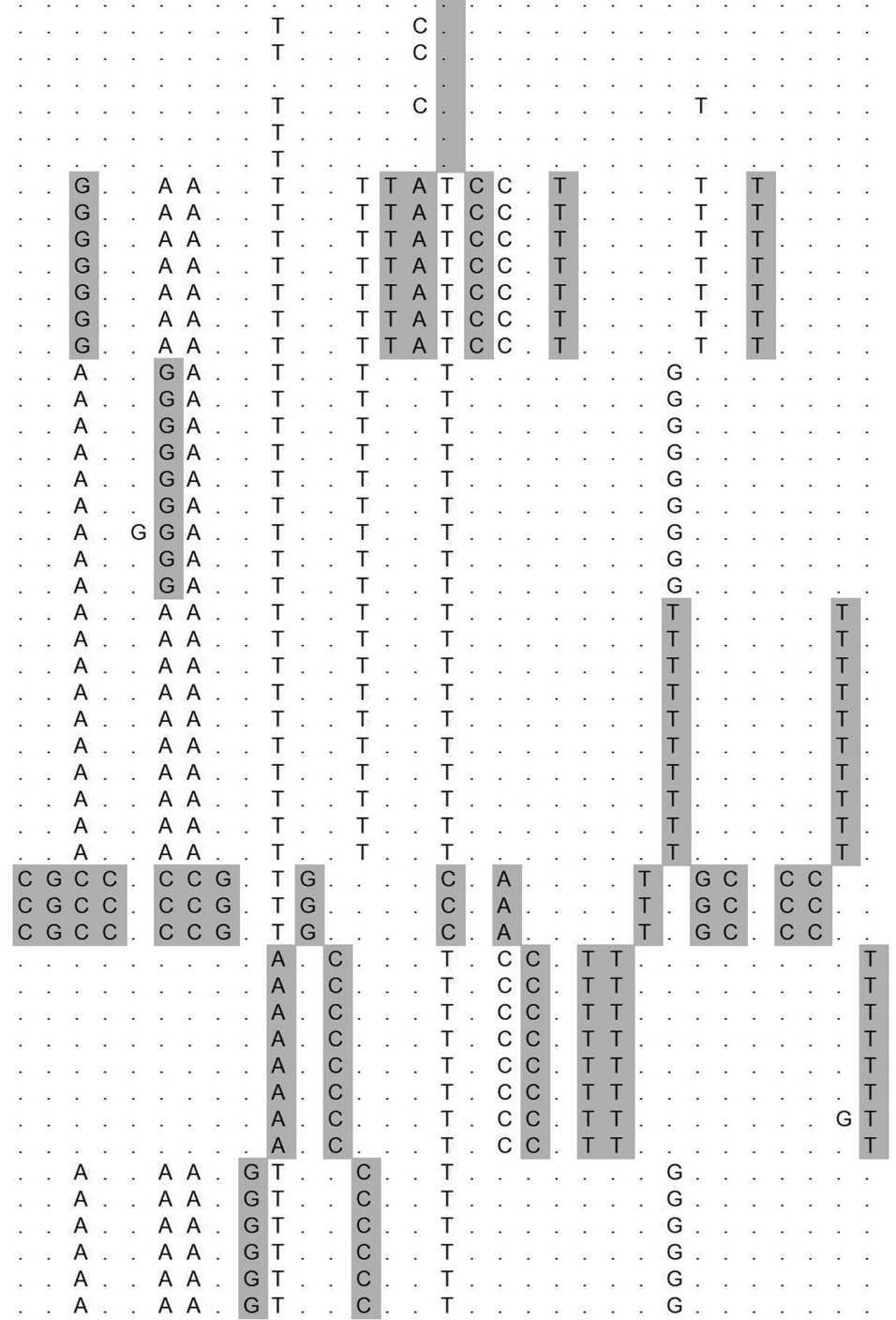

Figure 2. Diagnostic nucleotide sites in the alignment of $C O I$ sequences of the riverata species group. Numbers at the top show the positions of the sites in the COI alignment (584-687 bp in length). Shaded sites are diagnostic for each species. Dashes (-) indicate missing data.

\section{Taxonomy}

\section{Scaptodrosophila riverata species group}

Diagnosis. Body mostly yellow to yellowish brown, without patches; arista with two dorsal branches and one ventral branch in addition to the terminal bifurcation; facial 


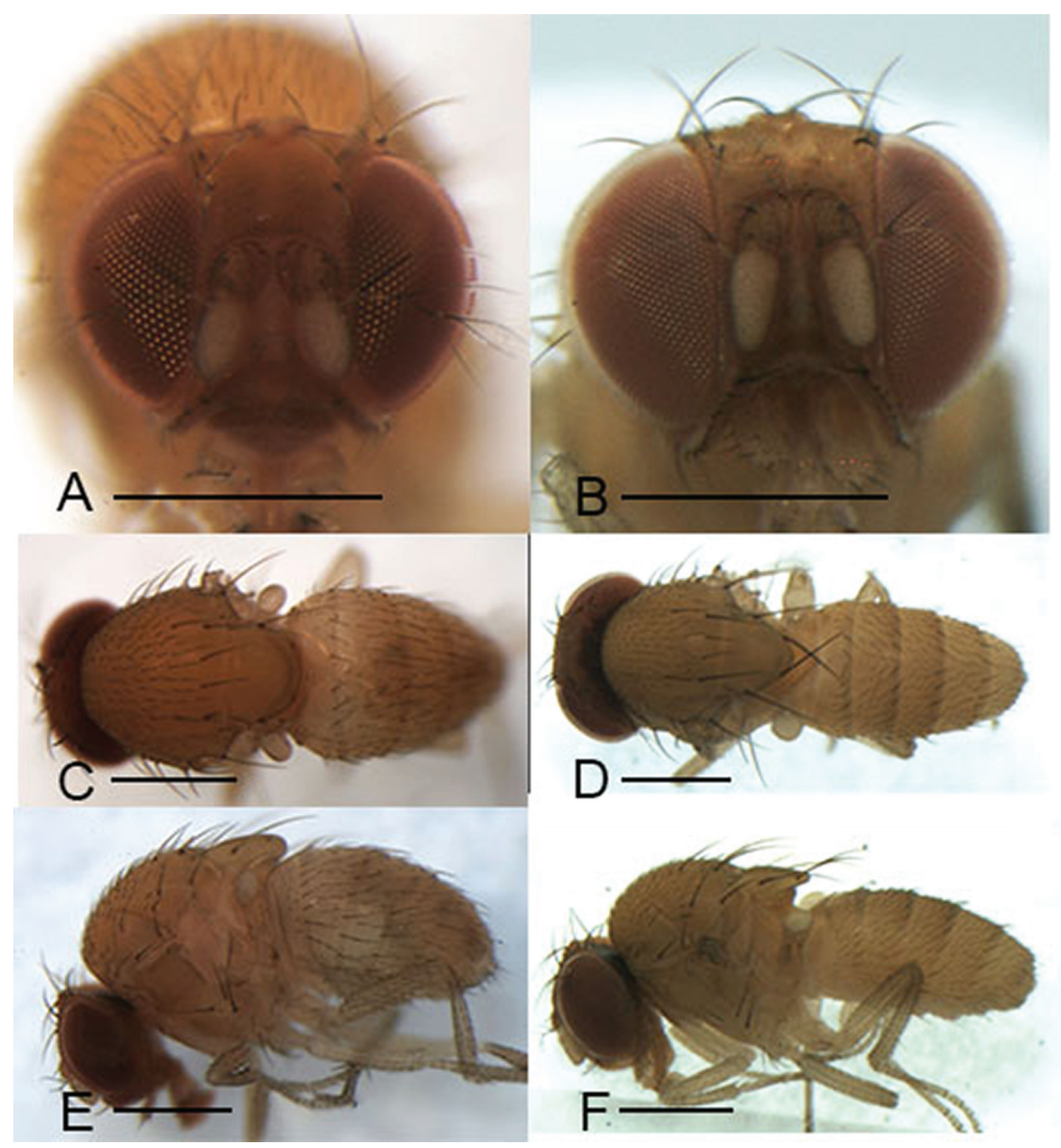

Figure 3. Head, mesonotum, scutellum, pleura and abdomen of male. A, C, E S. riverata (Singh \& Gupta, 1977) B, D, F S. puncticeps (Okada, 1956). Scale bars: $0.5 \mathrm{~mm}$.

carina large; prescutellar setae usually large, as long as anterior dorsocentral setae; hypandrium usually with a pair of long paramedian setae.

Description. Male and female: Head (Figs 3, 4, 5A, B, 6A): Eyes red. Ocellar triangle yellowish brown, with 4-6 setae above ocellar setae. Frons about 1/3 width of head, largely yellowish brown to brown, with a few minute setulae medially. Fronto-orbital pale yellowish brown; anterior reclinate orbital seta usually lateral to and about $1 / 3$ length of proclinate orbital seta; posterior reclinate orbital seta larger than other two orbital setae. Pedicel yellowish brown to brown, with a few of fine setae; first flagellomere pale yellowish. Face yellowish brown. Clypeus mostly yellow. Vibrissa prominent; subvibrissal setae small. Gena and postgena narrow, yellowish brown. Palpus yellow to yellowish brown with several setae. 

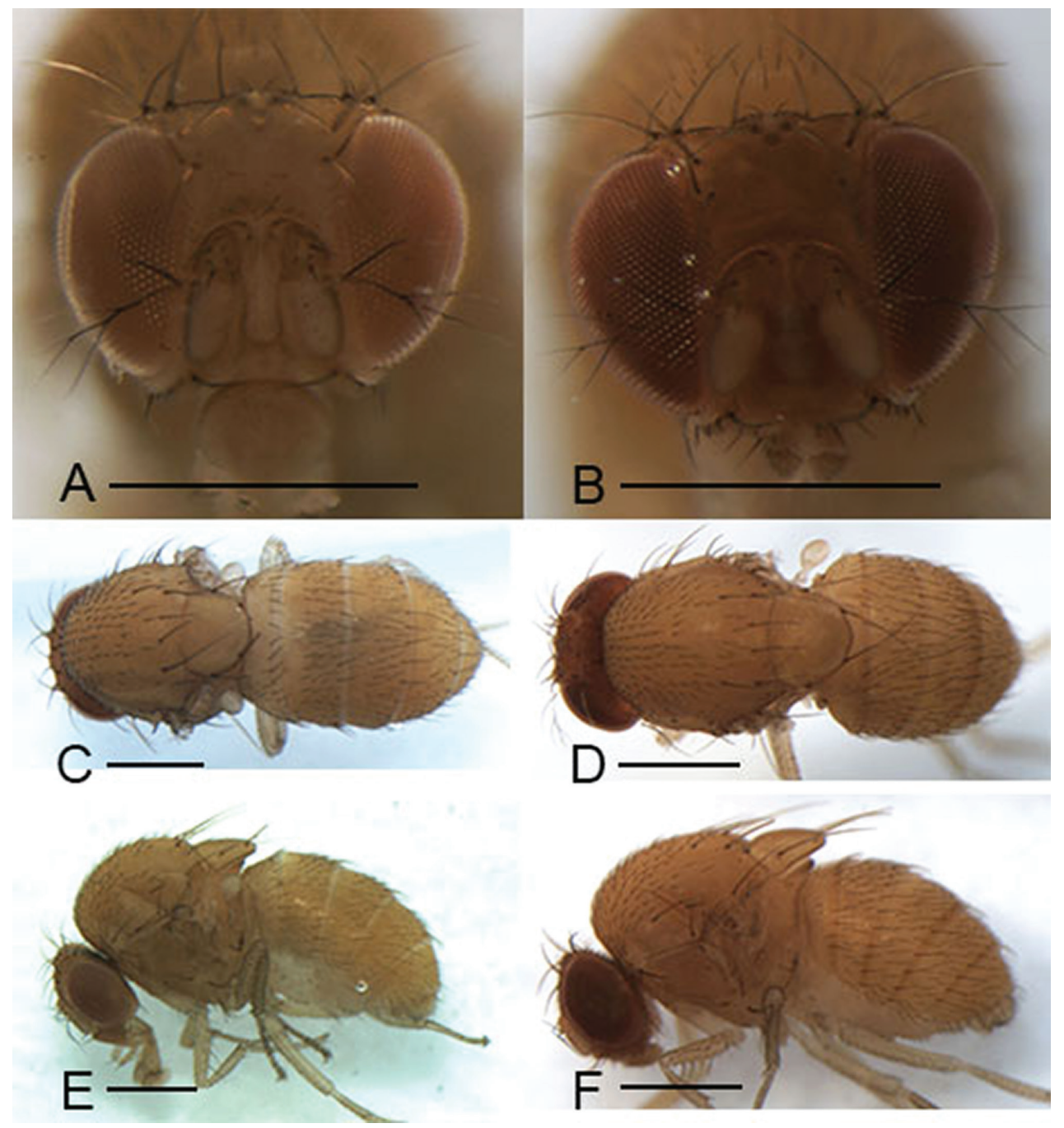

Figure 4. Head, mesonotum, scutellum, pleura and abdomen of male. A, C, E S. abdentata sp. nov. B, D, F S. platyrhina sp. nov. Scale bars: $0.5 \mathrm{~mm}$.

Thorax (Figs 3, 4, 5C-F, 6B, C): Mesonotum yellow to yellowish brown uniformly. Postpronotal lobe yellow with 2-3 long setae, and a few of shorter setae. Acrostichal setulae in ca 8-10 regular rows. Prescutellar setae usually as long as anterior dorsocentral setae. Pleura yellow to yellowish brown uniformly. Katepisternal setae usually subequal. Wing hyaline, sometimes infuscate, lacking patch. Basal medial-cubital crossvein absent. $\mathrm{R}_{4+5}$ nearly parallel distally with $\mathrm{M}_{1}$. Halter pale yellowish. Legs mostly yellow.

Abdomen (Figs 3, 4, 5C-F, 6B, C): Tergites yellow to yellowish brown, lacking patches.

Male terminalia (Figs 7-12, 13A-D): Epandrium pubescent, with several setae near posterior margin and ventral corner on each side. Surstylus usually with several 

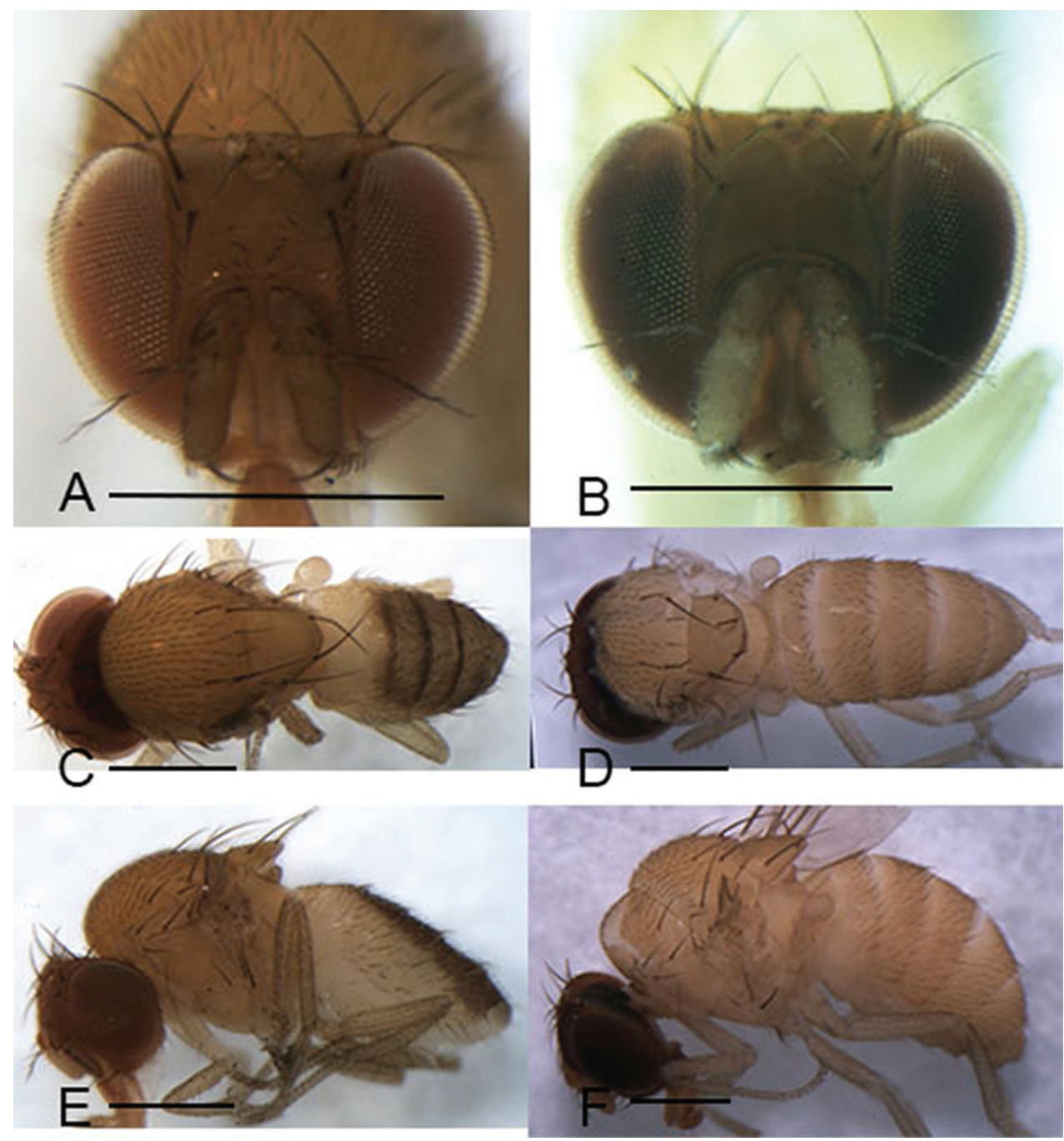

Figure 5. Head, mesonotum, scutellum, pleura and abdomen of male. A, C, E S. serrateifoliacea sp. nov. B, D, F S. sinuata sp. nov. Scale bars: $0.5 \mathrm{~mm}$.

peg-like prensisetae on caudal margin, and several setae on outer and inner surface. Hypandrium shallow, usually with a couple of paramedian setae distally. Cercus separated from epandrium, pubescent and setigerous. Paramere developed, with few sensillae. Gonopods (as dorsal extension of the hypandrium, see Ashley and Sinclair 2017) fused with each other, broadened to hood-shaped. Aedeagus bifid, glabrous.

Female terminalia (Figs 7-10, 11E, 13E): Oviscapt mostly yellowish brown to brown, broadened subapically.

In the following descriptions, only those characters differing from the above description were provided. 

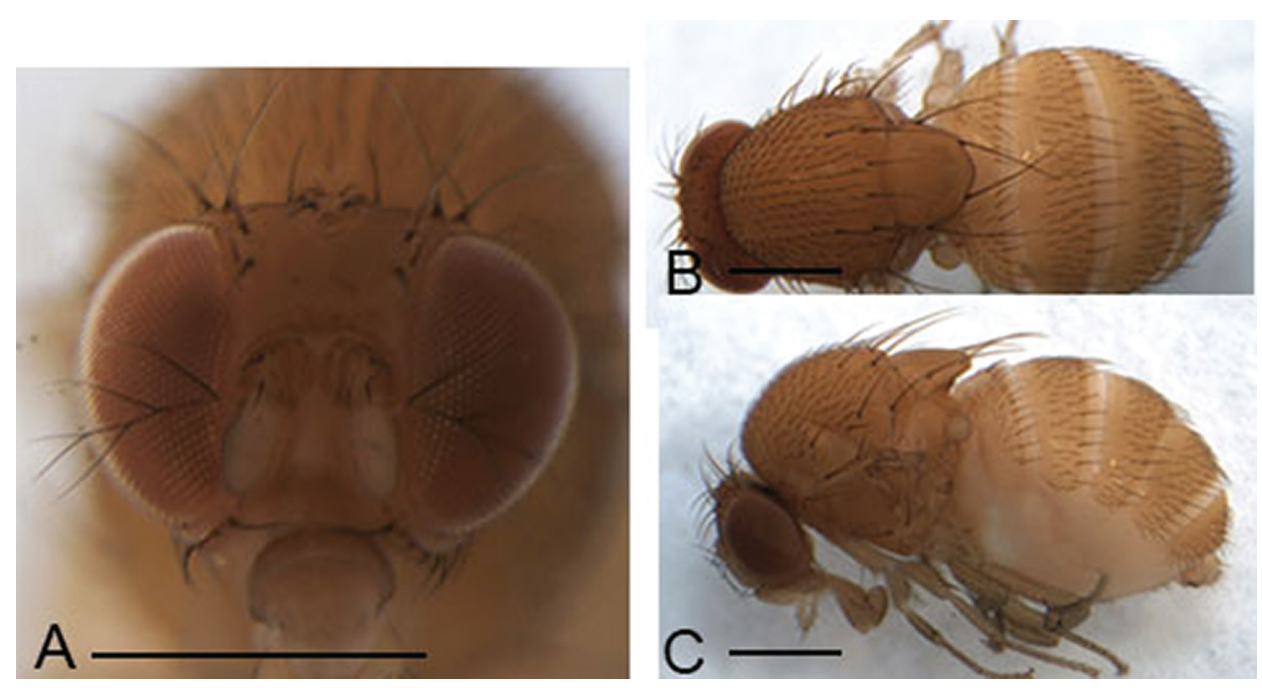

Figure 6. Head, mesonotum, scutellum, pleura and abdomen of male. A-C S. tanyrhina sp. nov. Scale bars: $0.5 \mathrm{~mm}$.

\section{Scaptodrosophila riverata (Singh \& Gupta, 1977)}

Figs 3A, C, E, 7

Drosophila riverata Singh \& Gupta, 1977: 240.

Material examined. China: $2 \widehat{\jmath}$ (SCAU, Nos 126828, 29), Banli, Chongzuo, Guangxi, alt. 160 m, 21.viii.2004, HW Chen; 1 ㅇ (SCAU, No. 126830), Ailaoshan, Jingdong, Yunnan, $4^{\circ} 32^{\prime} \mathrm{N}, 101^{\circ} 00^{\prime} \mathrm{E}$, alt. $2100 \mathrm{~m}$, 21.vi.2009, T Li; $3{ }^{\widehat{ }}$ (SCAU, Nos $110913-$ 15), Likan, Ximeng, Yunnan, $22^{\circ} 39^{\prime} \mathrm{N}, 99^{\circ} 36^{\prime} \mathrm{E}$, alt. $844 \mathrm{~m}$, 1.v.2016, YQ Liu; $10^{\wedge}$, 1 우 (SCAU, Nos 110916, 17), Huanglianshan, Lüchun, Yunnan, $23^{\circ} 20^{\prime} \mathrm{N}, 102^{\circ} 23^{\prime} \mathrm{E}$, alt. 600 m, 28.x.2018, HW Chen.

Diagnosis. Surstylus bifid, the upper lobe with about 5 thin, peg-like prensisetae and 1 fine seta, the below one with 1 thin, peg-like prensiseta and 2 fine setae (Fig. 7A, B); gonopods undeveloped (Fig. 7C, D).

Description. $(\widehat{O}$,, ) Head (Fig. 3A): Frons yellowish brown. Pedicel and first flagellomere maple. Facial carina yellowish, short, 1/3 length as face.

Thorax (Fig. 3C, E): Mesonotum and scutellum yellowish. Acrostichal setulae in ca 8 regular rows. Wings hyaline.

Abdomen (Fig. 3C, E): Tergites and sternites yellowish.

Male terminalia: Epandrium with ca 14 setae near posterior margin and ventral corner on each side (Fig. 7A, B). Hypandrium glabrous (Fig. 7C, D). Paramere slender and curved apically, with 3 sensillae subbasally (Fig. 7C, D).

Female terminalia (Fig. 7E): Oviscapt with 2 long subapical trichoid ovisensilla, 17 peg-like ovisensilla on each side on ventral margins and 5 trichoid ovisensilla on dorsal. 


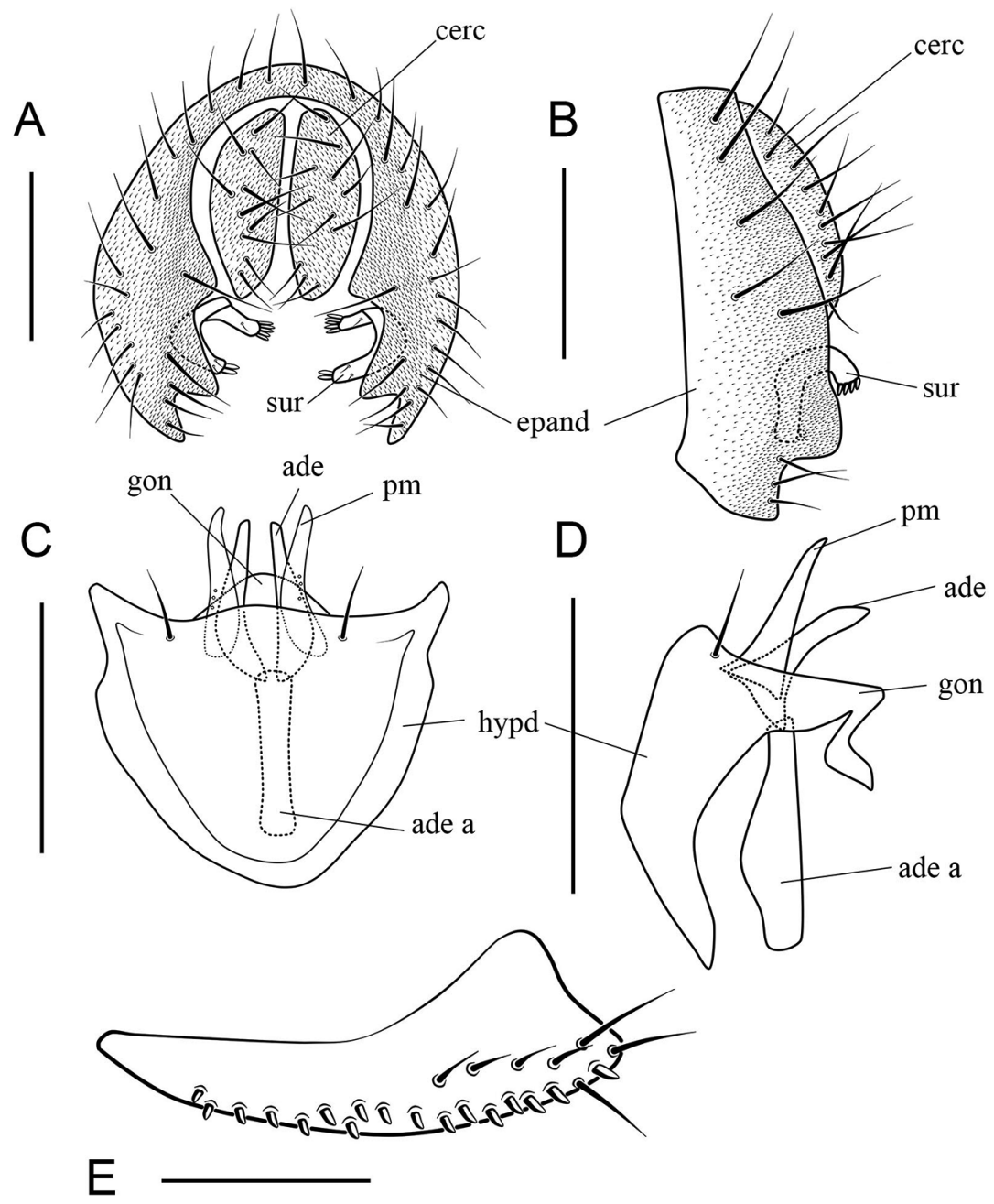

Figure 7. Scaptodrosophila riverata (Singh \& Gupta, 1977) đ. A Epandrium, surstylus and cercus (posterior view) B surstylus (lateral view) C hypandrium, aedeagus, aedeagal apodeme, paramere and gonopods (ventral view) D hypandrium, aedeagus, aedeagal apodeme, paramere and gonopods (lateral view) $\mathbf{E}$ oviscapt (lateral view). Scale bars: $0.1 \mathrm{~mm}$.

Measurements (range in $2 \widehat{\jmath}, 1$, in $\mathrm{mm}): \mathrm{BL}=(1.73-2.07,1.93 \mathrm{~mm}), \mathrm{ThL}=$ $(0.80-1.07,0.80), \mathrm{WL}=(1.80-2.27,1.93), \mathrm{WW}=(0.67-0.93,0.80)$.

Indices: $\mathrm{arb}=2 / 1$, avd $=0.60-1.00, \mathrm{adf}=2.00-2.50, \mathrm{flw}=2.00, \mathrm{FW} / \mathrm{HW}=$ $0.45-0.48, \mathrm{ch} / \mathrm{o}=0.08$, prorb $=0.80-0.83, \mathrm{rcorb}=0.40-0.60, \mathrm{vb}=0.33-0.50, \mathrm{dcl}=$ $0.54-0.60$, presctl $=0.45-0.60$, sctl $=1.00-1.10$, sterno $=0.67-0.75$, orbito $=0.67-$ $0.75, \mathrm{dcp}=0.30-0.40, \mathrm{sctlp}=0.83, \mathrm{C}=2.42-2.92,4 \mathrm{c}=1.00-1.20,4 \mathrm{v}=2.17-2.60$, $5 \mathrm{x}=1.50-2.00, \mathrm{ac}=2.40-3.00, \mathrm{M}=0.67-0.80, \mathrm{C} 3 \mathrm{~F}=0.25-0.33$.

Distribution. China (Guangxi, Yunnan), India, Myanmar. 


\section{Scaptodrosophila puncticeps (Okada, 1956)}

Figs 3B, D, F, 8

Drosophila puncticeps Okada, 1956: 94.

Material examined. China: 60, 5 q (SCAU, Nos 126796-804, 830), Shennongjia, Hubei, $31^{\circ} 49^{\prime} \mathrm{N}, 109^{\circ} 41^{\prime} \mathrm{E}$, alt. 1900 m, 31.vii. 2004, 6.viii.2005, HW Chen, HZ Cao; $1 \delta^{\top}$ (SCAU, No. 126831), Tianmushan, Linan, Zhejiang, $30^{\circ} 20^{\prime} \mathrm{N}, 119^{\circ} 25^{\prime} \mathrm{E}$, alt. 800 m, 30.vi.-2.viii.2011, tussocks, ZF Shao, SJ Yan; $1 \overbrace{}^{\Uparrow}$ (SCAU, No. 126825), Miyaluo, Aba, Sichuan, alt. 2650 m, 14.ix.2005, MF Xu.

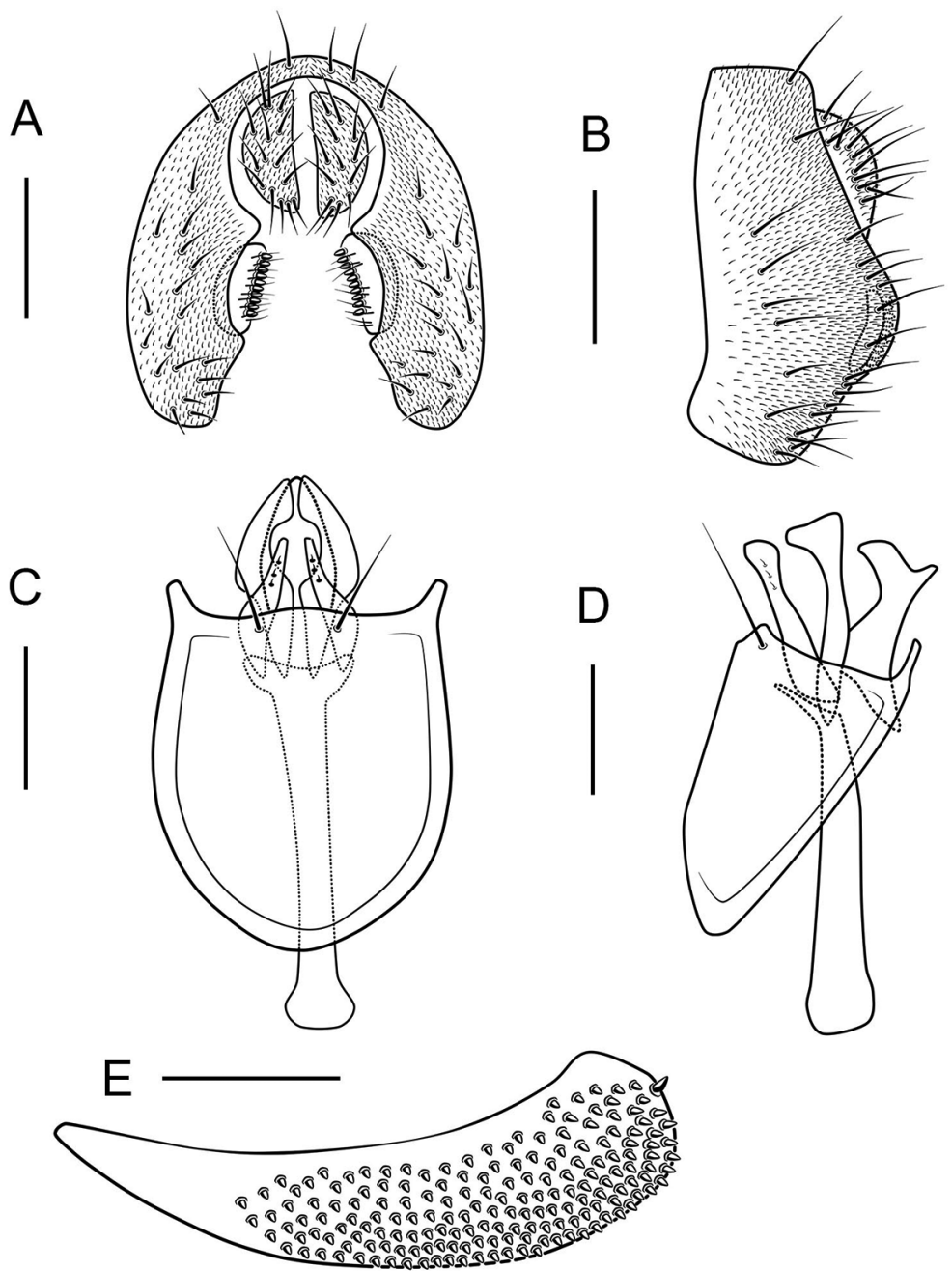

Figure 8. Scaptodrosophila puncticeps (Okada, 1956) D. A Epandrium, surstylus and cercus (posterior view) B epandrium, surstylus and cercus (lateral view) C hypandrium, aedeagus, aedeagal apodeme, paramere and gonopods (ventral view) D hypandrium, aedeagus, aedeagal apodeme, paramere and gonopods (lateral view) E oviscapt (lateral view). Scale bars: $0.1 \mathrm{~mm}$. 
Diagnosis. Clypeus reddish brown; palpus pale yellow; gonopods roundly expanded in lateral view. (Fig. 7C, D).

Description. $(\hat{O}$, + ) Head (Fig. 3B): Frons reddish brown. Pedicel yellowish brown. Facial carina creamy white, short, 1/3 length as face.

Thorax (Fig. 3D, F): Mesonotum and scutellum yellow. Acrostichal setulae in ca 7 irregular rows. Halter yellowish brown. Wing greyish.

Abdomen (Fig. 3D, F): Tergites yellowish brown.

Male terminalia: Epandrium with ca 21 setae near posterior margin and ventral corner on each side (Fig. 8A, B). Surstylus small with 10 peg-like prensisetae on caudal margin, and ca 16 setae on outer and inner surface (Fig. 8A, B). Hypandrium pubescent near paramedian setae (Fig. 8C, D). Paramere broadened medially with ca 3 sensilla (Fig. 8C, D). Aedeagus broadened apically, curved medially, sickle-shaped anteriorly in lateral view (Fig. 8C, D).

Female terminalia (Fig. 8E): Oviscapt densely covered with peg-like ovisensilla.

Measurements (range in $60^{\lambda}, 5$, , in $\mathrm{mm}$ ): $\mathrm{BL}=1.87-3.87,2.20-2.67, \mathrm{ThL}=$ $0.80-1.13,0.80-1.00, \mathrm{WL}=2.13-2.93,2.13-2.73$, WW $=0.73-1.20,0.86-1.07$.

Indices: $\mathrm{arb}=2 / 1$, avd $=0.25-1.00$, adf $=1.50-2.00, \mathrm{flw}=2.00-4.00, \mathrm{FW} / \mathrm{HW}=$ $0.41-0.50, \mathrm{ch} / \mathrm{o}=0.07-0.13$, prorb $=0.71-1.00, \mathrm{rcorb}=0.29-0.50, \mathrm{vb}=0.33-0.75$, $\mathrm{dcl}=0.45-0.70$, presctl $=0.33-0.57$, sctl $=0.85-1.22$, sterno $=0.30-0.67$, orbito $=$ $0.50-0.75, \mathrm{dcp}=0.44-0.56$, sctlp $=0.71-1.25, \mathrm{C}=2.75-4.00,4 \mathrm{c}=0.60-0.92,4 \mathrm{v}=$ $1.40-2.08,5 \mathrm{x}=1.20-1.67, \mathrm{ac}=0.86-2.33, \mathrm{M}=0.40-0.60, \mathrm{C} 3 \mathrm{~F}=0.30-0.38$.

Distribution. China (Zhejiang, Hubei, Hunan, Sichuan, Guizhou, Yunnan), Kuril Islands, Korea, Japan (Kanto).

\section{Scaptodrosophila abdentata sp. nov.}

http://zoobank.org/426657F1-40A2-4E11-BFB1-BBCB8B7A2D42

Figs 4A, C, E, 9

Material examined. Holotype. ChIna: $\widehat{~}$ (SCAU, No. 127162), Nanling, Shaoguan, Guangdong, $24^{\circ} 38^{\prime} \mathrm{N}, 112^{\circ} 40^{\prime} \mathrm{E}$, alt. 800 m, 3.iii.2004, MF Xu. Paratypes. China: $10^{\lambda}, 2$ ㅇ (SCAU, Nos 127163-65), Nanling, Ruyuan, Guangdong, $24^{\circ} 38^{\prime} \mathrm{N}$, $112^{\circ} 40^{\prime} \mathrm{E}$, alt. $800 \mathrm{~m}$, 3.iii.2004, MF Xu; 10, 2 + (SCAU, Nos 127166-68), Jianfengling, Ledong, Hainan, $18^{\circ} 41^{\prime} \mathrm{N}, 108^{\circ} 52^{\prime} \mathrm{E}$, alt. 700 m, 23.iv.2007, HW Chen;

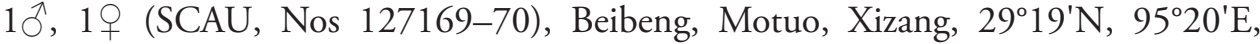
alt. 780 m, 4.x.2010, YR Su; $2 \hat{\jmath}$ (SCAU, Nos 127171-72), Wangtianshu, Mengla, Yunnan, $21^{\circ} 28^{\prime} \mathrm{N}, 101^{\circ} 38^{\prime} \mathrm{E}$, alt. $580 \mathrm{~m}$, 23.iv.2007, HW Chen; $10^{\wedge}$ (SCAU, No. 110918), Muyiji, Ximeng, Yunnan, $22^{\circ} 37^{\prime}$ N, $99^{\circ} 35^{\prime}$ E, alt. 1100 m, 23.iv.2007, HW Chen; $1 \widehat{\partial}^{\hat{\gamma}}$ (SCAU, No. 110919), Muyiji, Ximeng, Yunnan, $22^{\circ} 37^{\prime} \mathrm{N}, 99^{\circ} 35^{\prime} \mathrm{E}$, alt. 1203 m, 30.iv. 2016, YQ Liu; $2 \hat{\jmath}$ (SCAU, Nos 110920, 21), Mengdong, Cangyuan, Yunnan, $23^{\circ} 10^{\prime} \mathrm{N}, 99^{\circ} 13^{\prime} \mathrm{E}$, alt. $1323 \mathrm{~m}$, 6.v.2016, YQ Liu.

Diagnosis. Surstylus with a row of ca 9 long, peg-like prensisetae on caudal margin, and ca 6 setae on outer surface (Fig. 9A, B); paramere leaf-shaped in lateral view, with ca 3 sensillae and a row of fine setae (Fig. 9C, D); aedeagus broadened apically (Fig. 9C, D). 


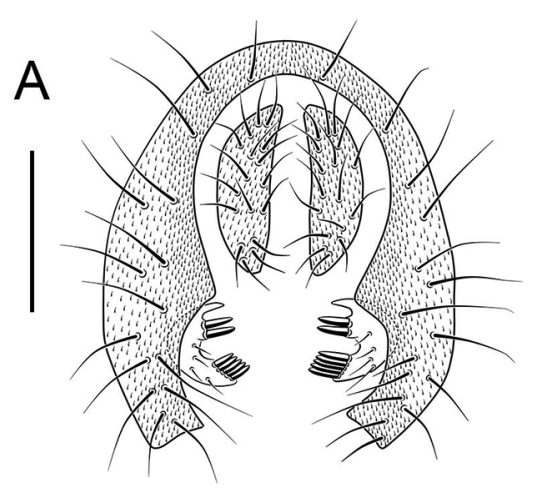

B
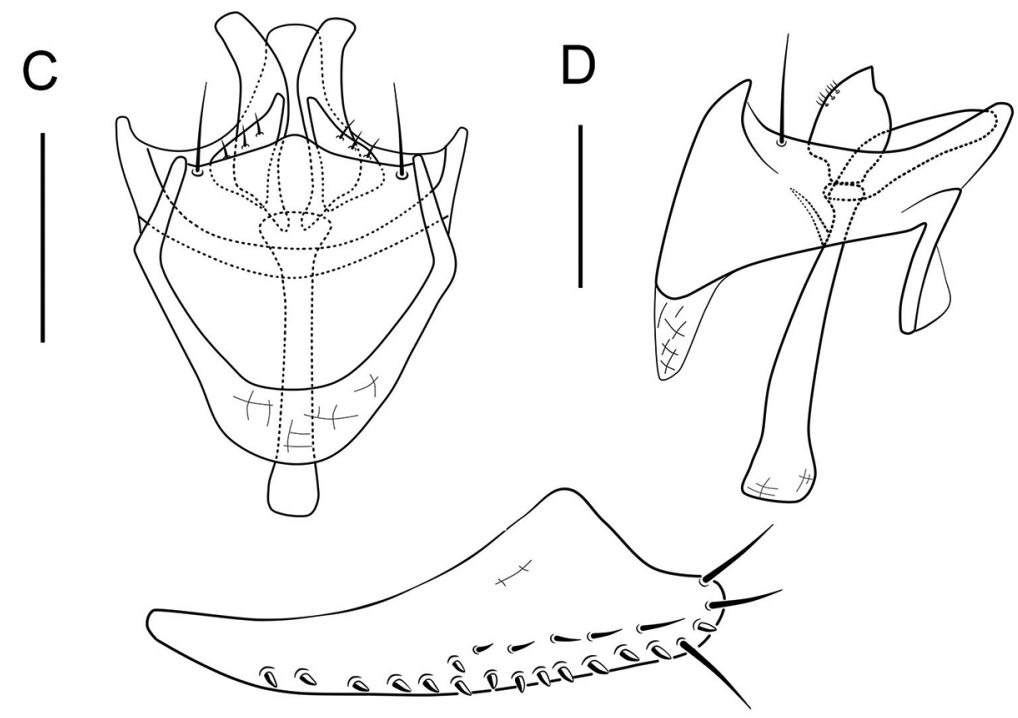

\section{$\mathrm{E}$}

Figure 9. Scaptodrosophila abdentata sp. nov. O. A Epandrium, surstylus and cercus (posterior view) B epandrium, surstylus and cercus (lateral view) $\mathbf{C}$ hypandrium, aedeagus, aedeagal apodeme, paramere and gonopods (ventral view) D hypandrium, aedeagu, aedeagal apodeme, paramere and gonopods (lateral view) E oviscapt (lateral view). Scale bars: $0.1 \mathrm{~mm}$.

Description. ( ${ }^{\lambda}$, , $)$ Head (Fig. 4A): Frons yellowish brown. Pedicel brown. First flagellomere yellowish. Facial carina brownish, short, 1/3 length as face.

Thorax (Fig. 4C, E): Mesonotum and scutellum yellowish brown. Acrostichal setulae in ca 8 regular rows. Halter hazel. Wings yellowish.

Abdomen (Fig. 4C, E): Tergites creamy white.

Male terminalia: Epandrium with ca 15 setae near posterior margin and ventral corner on each side (Fig. 9A, B). Hypandrium glabrous (Fig. 9C, D).

Female terminalia (Fig. 8E): Oviscapt with 3 long subapical trichoid ovisensilla, 15 peg-like ovisensilla on each side on ventral margins and 5 trichoid ovisensilla on dorsal. 
Measurements [holotype $\widehat{\partial}$ (range in $5 \hat{\jmath}, 59)$, in $\mathrm{mm}$ ]: $\mathrm{BL}=2.20(1.73-1.93$, 2.00-2.27), ThL $=1.07$ (0.80-1.00, 1.00-1.13), $\mathrm{WL}=2.40(1.87-2.20,2.20-2.33)$, $\mathrm{WW}=0.93(0.67-0.87,0.87-0.93)$.

Indices: $\mathrm{arb}=2 / 1(2 / 1)$, avd $=0.60(0.60-1.00), \mathrm{adf}=2.50(2.00-2.50), \mathrm{flw}=$ $2.00(1.50-2.50), \mathrm{FW} / \mathrm{HW}=0.39(0.41-0.50), \mathrm{ch} / \mathrm{o}=0.07(0.08-0.10)$, prorb $=$ $0.83(0.71-1.00), \mathrm{rcorb}=0.50(0.33-0.50), \mathrm{vb}=0.50(0.50-0.67), \mathrm{dcl}=0.58(0.54-$ $0.75)$, presctl $=0.50(0.38-0.55)$, sctl $=1.25(1.00-1.31)$, sterno $=0.78(0.60-0.72)$, orbito $=0.67(0.67), \mathrm{dcp}=0.33(0.31-0.44), \mathrm{sctlp}=1.83(0.80-1.00), \mathrm{C}=3.23$ $(2.21-3.00), 4 \mathrm{c}=0.93(0.92-1.20), 4 \mathrm{v}=2.43(2.21-3.00), 5 \mathrm{x}=1.60(1.60-2.00)$, $\mathrm{ac}=2.60(2.40-3.00), \mathrm{M}=0.57(0.57-0.82), \mathrm{C} 3 \mathrm{~F}=0.38(0.31-0.38)$.

Etymology. A combination of the Latin words: "ab-" + "dentatus", referring to the surstylus with a line discontinuous of prensisetae.

Distribution. China (Guangdong, Hainan, Yunnan, Xizang).

\section{Scaptodrosophila platyrhina sp. nov.}

http://zoobank.org/E1397E1F-B748-41DE-B651-B8727E27AD34

Figs 4B, D, F, 10

Material examined. Holotype. China: $\widehat{\partial}$ (SCAU, No. 126838), Menglun, Mengla, Yunnan, $21^{\circ} 41^{\prime} \mathrm{N}, 101^{\circ} 25^{\prime} \mathrm{E}$, alt. $680 \mathrm{~m}, 17 . \mathrm{iv}$. 2007, HW Chen. Paratypes. China: $4{ }^{\top}, 7$ ㅇ (SCAU, Nos 126839-47), Menglun, Mengla, Yunnan, $21^{\circ} 41^{\prime} \mathrm{N}, 101^{\circ} 25^{\prime} \mathrm{E}$,

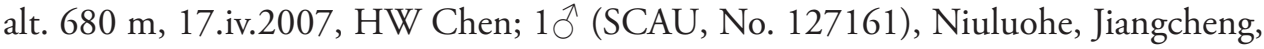
Yunnan, alt. $800 \mathrm{~m}, 22^{\circ} 30^{\prime} \mathrm{N}, 1^{\circ} 1^{\circ} 34^{\prime} \mathrm{E}, 21 . i v .2010$, HW Chen; $10^{\wedge}$ (SCAU, No. 110923), Guanlei, Mengla, Yunnan, $21^{\circ} 38^{\prime} \mathrm{N}, 101^{\circ} 10^{\prime} \mathrm{E}$, alt. $620 \mathrm{~m}$, 21.iv.2016, YQ Liu; $2 \hat{O}, 2$ (SCAU, Nos 110924, 26-28), Menglun, Mengla, Yunnan, $24^{\circ} 41^{\prime} \mathrm{N}$, $101^{\circ} 25^{\prime} \mathrm{E}$, alt. $554 \mathrm{~m}, 12 . \mathrm{iv} .2010$, HW Chen.

Diagnosis. Facial carina short; paramere finger-like in lateral view, with ca 4 sensillae basally (Fig. 10C, D); aedeagus bifid apically (Fig. 10C, D); gonopods undeveloped (Fig. 10C, D).

Description. Head (Fig. 4B): Frons yellowish brown. Pedicel yellowish brown. First flagellomere yellowish. Facial carina hazel, short, 1/3 length as face.

Thorax (Fig. 4D, F): Mesonotum and scutellum yellowish. Acrostichal setulae in ca 8 regular rows. Halter hazel. Wings yellowish.

Abdomen (Fig. 4D, F): Tergites yellowish.

Male terminalia: Epandrium with ca 11 setae near posterior margin and ventral corner per side (Fig. 10A, B). Surstylus with ca 10 long, peg-like prensisetae on caudal margin, and ca 3 setae on outer surface (Fig. 10A, B). Hypandrium glabrous (Fig. 10C, D).

Female terminalia (Fig. 10E): Oviscapt with 3 long subapical trichoid ovisensilla, ca 21 peg-like ovisensilla on each side on ventral margins and 5 trichoid ovisensilla on dorsal.

Measurements [holotype $\hat{\sigma}$ (range in $5 \hat{\alpha}, 5$ + $)$, in $\mathrm{mm}$ ]: $\mathrm{BL}=2.27(2.00-2.33$, $1.93-2.13), \mathrm{THL}=1.13(0.80-1.07,1.00-1.07), \mathrm{WL}=2.47(2.20-2.40,2.20-2.40)$, WW $=0.93(0.80-0.93,0.80-0.93)$. 


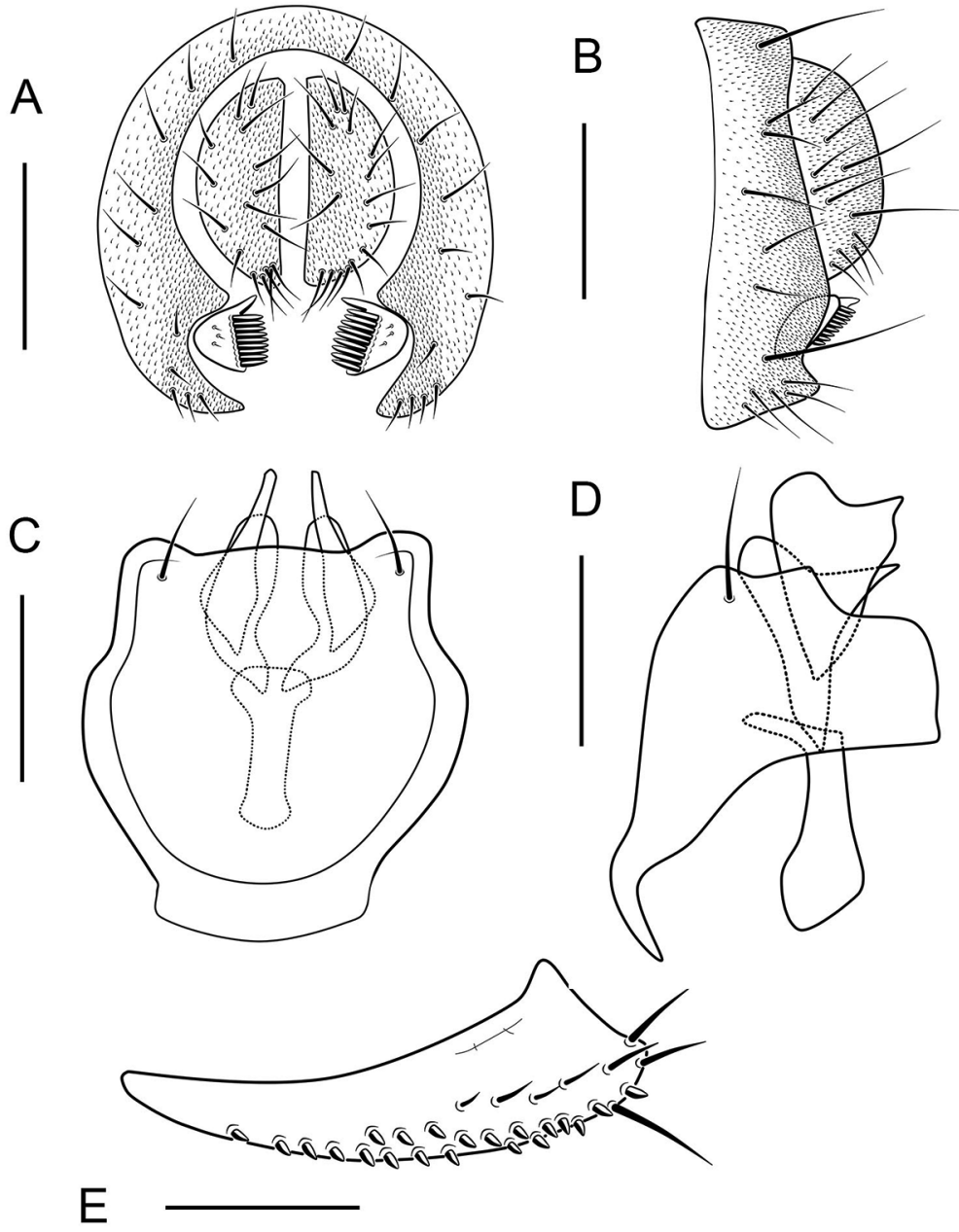

Figure 10. Scaptodrosophila platyrhina sp. nov. $\widehat{\partial}$. A Epandrium, surstylus and cercus (posterior view) $\mathbf{B}$ epandrium, surstylus and cercus (lateral view) $\mathbf{C}$ hypandrium, aedeagus, aedeagal apodeme, paramere and gonopods (ventral view) D hypandrium, aedeagus, aedeagal apodeme, paramere and gonopods (lateral view) E oviscapt (lateral view). Scale bars: $0.1 \mathrm{~mm}$.

Indices: $\mathrm{arb}=2 / 1(2 / 1)$, avd $=0.60(0.60-0.80), \mathrm{adf}=2.50(2.00-2.50), \mathrm{flw}=$ $2.00(2.00), \mathrm{FW} / \mathrm{HW}=0.43(0.41-0.45), \mathrm{ch} / \mathrm{o}=0.08(0.08-0.09)$, prorb $=0.83$ $(0.40-1.00), \mathrm{rcorb}=0.50(0.33-0.60), \mathrm{vb}=0.40(0.40-0.50), \mathrm{dcl}=0.71(0.50-0.73)$, presctl $=0.43(0.40-0.50)$, sctl $=1.00(0.85-1.27)$, sterno $=0.89(0.70-0.89)$, orbito $=0.67(0.67), \mathrm{dcp}=0.36(0.31-0.36), \mathrm{sctl} p=1.00(0.71-1.00), \mathrm{C}=2.86(2.64-$ $2.79), 4 \mathrm{c}=1.00(0.93-1.08), 4 \mathrm{v}=2.36(2.00-2.46), 5 \mathrm{x}=1.67(1.50-1.80), \mathrm{ac}=2.80$ (2.80-3.00), $\mathrm{M}=0.71(0.64-0.71), \mathrm{C} 3 \mathrm{~F}=0.36(0.29-0.36)$.

Etymology. A combination of the Latin words: "platys" + "rhinos", referring to the flat carina.

Distribution. China (Yunnan). 


\section{Scaptodrosophila serrateifoliacea sp. nov.}

http://zoobank.org/9DC99991-AE49-4AC7-854B-C6CBC6AC9E88

Figs $5 \mathrm{~A}, \mathrm{C}, \mathrm{E}, 11$

Material examined. Holotype. CHINA: $\lesssim$ (SCAU, No. 126826), Hesong, Menghai, Yunnan, $21^{\circ} 49^{\prime} \mathrm{N}, 100^{\circ} 06^{\prime}$ E, alt. 1900 m, 11.iv. 2011, JM Lu, YR Su, ZF Shao, SJ Yan. Paratypes. China: $10^{\top}$ (SCAU, No. 126827), Hesong, Menghai, Yunnan, $21^{\circ} 50^{\prime} \mathrm{N}, 100^{\circ} 06^{\prime} \mathrm{E}$, alt. 1900 m, 11.iv.2011, JM Lu, YR Su, ZF Shao, SJ Yan. 10̄ (SCAU, No. 110929),

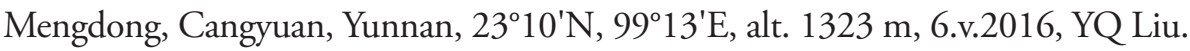
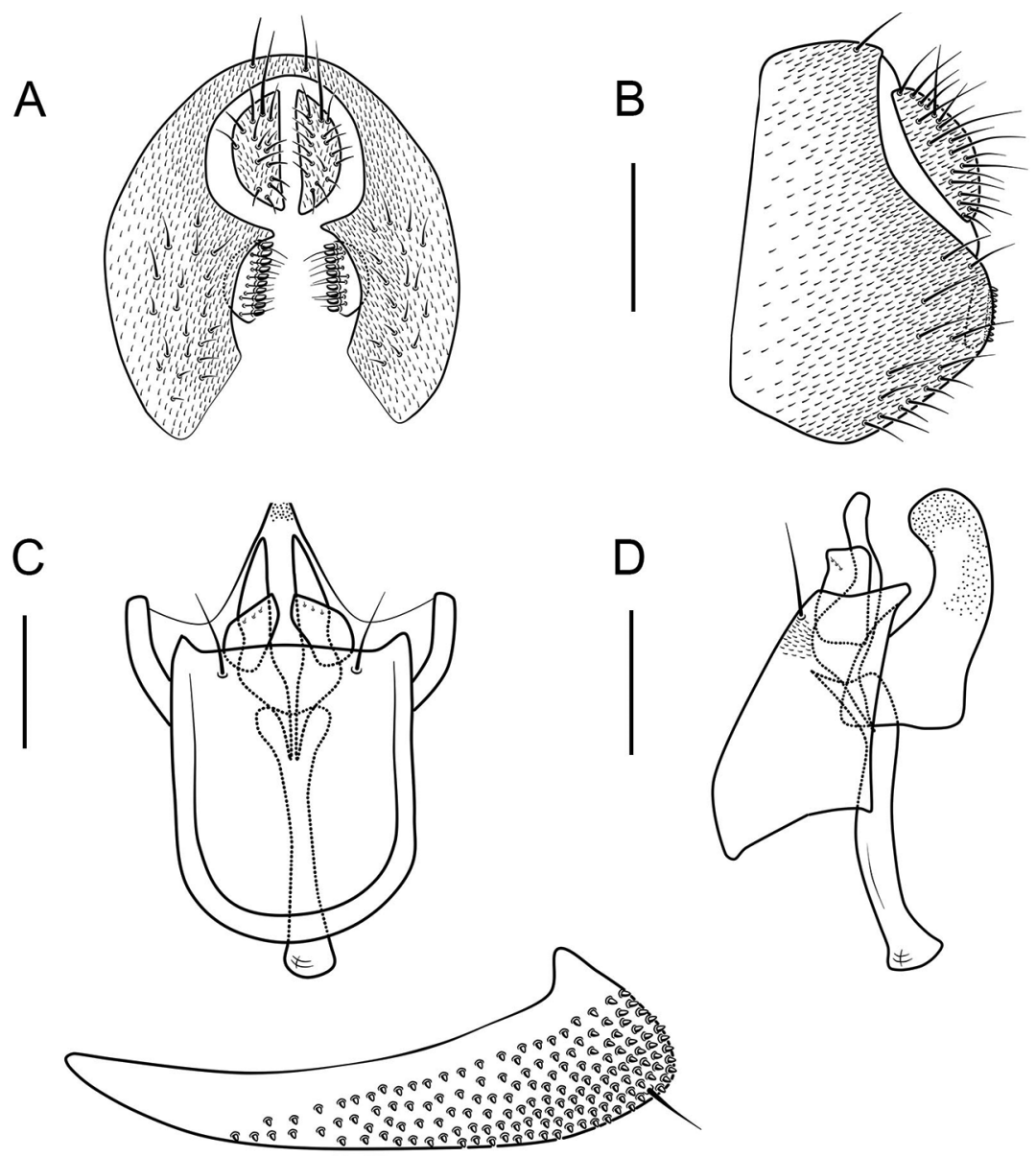

\section{$\mathrm{E}$}

Figure II. Scaptodrosophila serrateifoliacea sp. nov. $\widehat{\partial}$. A Epandrium, surstylus and cercus (posterior view) B epandrium, surstylus and cercus (lateral view) C hypandrium, aedeagus, aedeagal apodeme, paramere and gonopods (ventral view) D hypandrium, aedeagus, aedeagal apodeme, paramere and gonopods (lateral view) E oviscapt (lateral view). Scale bars: $0.1 \mathrm{~mm}$. 
Diagnosis. Paramere quadrangle-shaped in lateral view, with ca 3 sensillae medially (Fig. 11C, D); gonopods developed, arm-shaped, with many finely acanthoid processes (Fig. 11C, D); aedeagus beanpod-shaped in lateral view (Fig. 11C, D).

Description. ( $\hat{O}$, ㅇ) Head (Fig. 5A): Frons yellowish brown. Pedicel yellow. First flagellomere yellowish. Facial carina yellowish, short, 1/2 length as face.

Thorax (Fig. 5C, E): Mesonotum and scutellum dark brown. Acrostichal setulae in ca 8 irregular rows. Halter yellowish brown. Wings hazel.

Abdomen (Fig. 5C, E): First tergite and second tergite yellow. Third to sixth tergites brown.

Male terminalia: Epandrium with ca 19 setae near posterior margin and ventral corner on each side (Fig. 11A, B). Surstylus broad, with ca 10 fine peg-like prensisetae on caudal margin, and numerous setae on outer and inner surfaces (Fig. 11A, B). Hypandrium pubescent near paramedian setae (Fig. 11C, D).

Female terminalia (Fig. 11E): Oviscapt with 1 long subapical trichoid ovisensillum on ventral margins and densely covered with peg-like ovisensilla.

Measurements [holotype $\hat{\partial}$ (range in $1 \hat{\partial}, 1$ ) $)$, in $\mathrm{mm}$ ]: $\mathrm{BL}=1.93(2.22,1.80)$, $\mathrm{ThL}=0.93(0.889,1.00), \mathrm{WL}=2.13(2.44,2.13), \mathrm{WW}=0.87(0.978,0.93)$.

Indices: $\mathrm{arb}=2 / 1(2 / 1)$, avd $=0.67(0.67-0.73)$, $\mathrm{adf}=1.00(1.09-1.50), \mathrm{fl}=$ $1.33(1.59-2.50), \mathrm{FW} / \mathrm{HW}=0.45(0.344-0.48), \mathrm{ch} / \mathrm{o}=0.15(0.11-0.13)$, prorb $=$ $1.00(0.71-1.40), \mathrm{rcorb}=0.40(0.20-0.43), \mathrm{vb}=0.50(0.59-0.67), \mathrm{dcl}=0.50(0.59-$ $0.64)$, presctl $=0.33(0.36-0.37)$, sctl $=0.92(1.22-1.08)$, sterno $=0.50(0.33-0.45)$, orbito $=0.67(0.65-0.67), \mathrm{dcp}=0.44(0.42-0.44), \mathrm{sctl} p=0.83(1.20-1.25), \mathrm{C}=2.92$ (2.93-4.30), $4 \mathrm{c}=0.81(0.55-0.82), 4 \mathrm{v}=1.88(1.66-1.76), 5 \mathrm{x}=1.75(0.94-1.33)$, ac $=2.60$ (1.59-2.33), $\mathrm{M}=0.44(0.34-0.47), \mathrm{C} 3 \mathrm{~F}=0.38(0.50-0.60)$.

Etymology. A combination of the Latin words "serratus" (= serrated) + "foliaceus" (= folium), referring to the gonopods with numerous finely serrated processes.

Distribution. China (Yunnan).

\section{Scaptodrosophila sinuata sp. nov.}

http://zoobank.org/972ED4E8-7F80-4F9C-9610-B9922E4EBF4B

Figs 5B, D, F, 12

Material examined. Holotype. CHINA: ${ }^{\lambda}$ (SCAU, No. 110939), Laobiangou, Benxi, Liaoning, $41^{\circ} 04^{\prime} \mathrm{N}, 124^{\circ} 30^{\prime} \mathrm{E}$, alt. 575 m, 21.vi.2018, Y Lin. Paratypes. China: $2{ }^{\jmath}$ (SCAU, Nos 110936, 37), Dayangcha, Kuangdian, Liaoning, $41^{\circ} 01^{\prime} \mathrm{N}, 124^{\circ} 37^{\prime} \mathrm{E}$. alt. 485 m, 23.vi.2018, L Gong; $2 \widehat{\gamma}$ (SCAU, Nos 110938, 40), Laobiangou, Benxi, Liaoning, $41^{\circ} 04^{\prime} \mathrm{N}, 124^{\circ} 30^{\prime} \mathrm{E}$, alt. $575 \mathrm{~m}, 21 . v i .2018$, Y Lin; $30^{\lambda}$ (SCAU, Nos

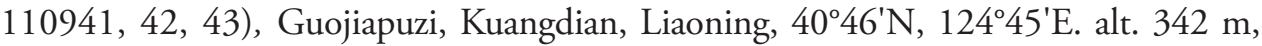
26.vi.2018, L Gong.

Diagnosis. Paramere quadrangle-shaped in lateral view, with ca 3 sensillae medially (Fig. 12A, B); gonopods large, with many finely acanthoid processes (Fig. 12C, D); aedeagus beanpod-shaped in lateral view (Fig. 12C, D). 


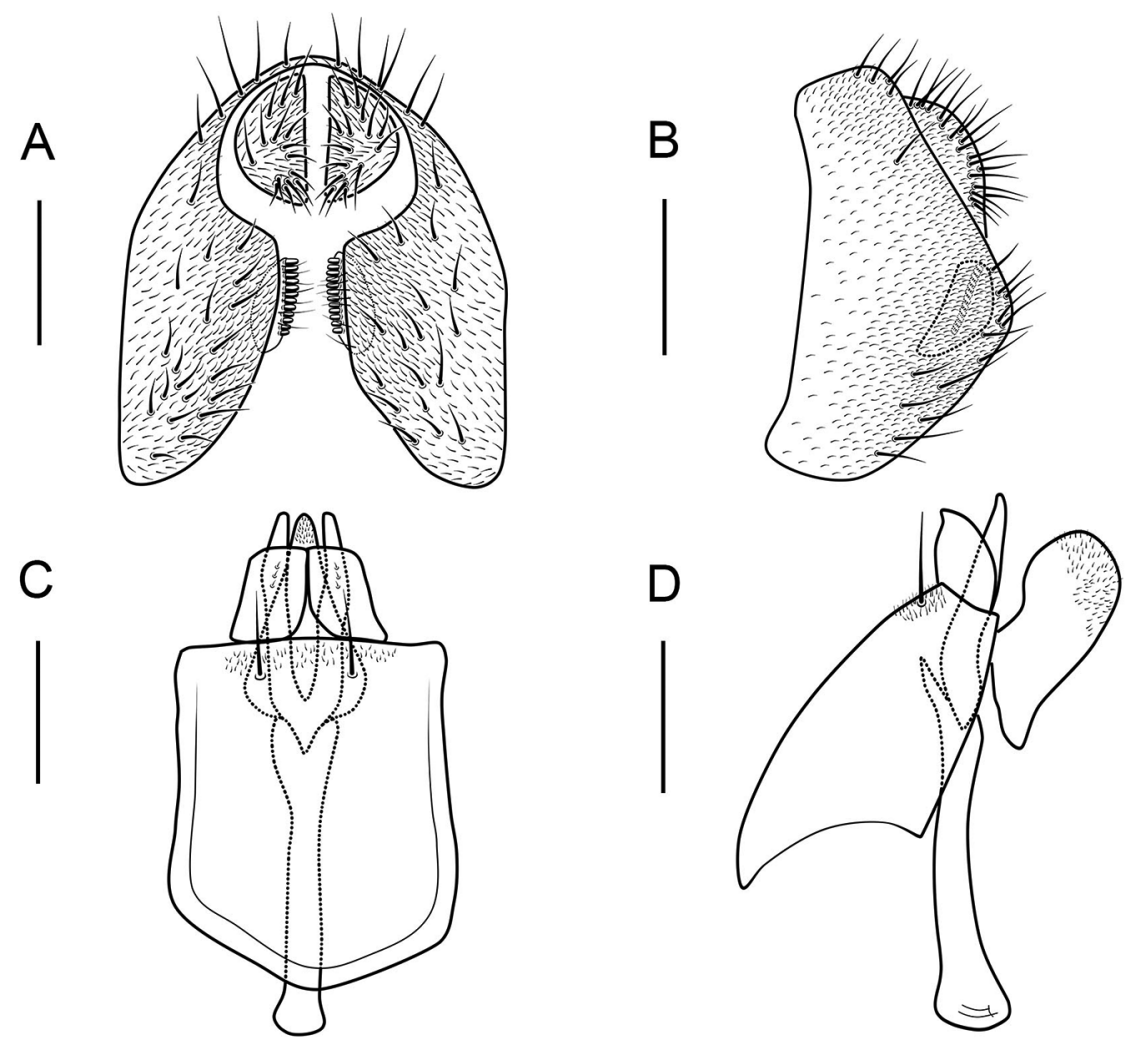

Figure 12. Scaptodrosophila sinuata sp. nov. O. A Epandrium, surstylus and cercus (posterior view) B epandrium, surstylus and cercus (lateral view) $\mathbf{C}$ hypandrium, aedeagus, aedeagal apodeme, paramere and gonopods (ventral view) D hypandrium, aedeagus, aedeagal apodeme, paramere and gonopods (lateral view). Scale bars: $0.1 \mathrm{~mm}$.

Description. ( $\overbrace{}^{\Uparrow})$ Head (Fig.5B): Frons yellowish brown. Pedicel yellow. First flagellomere yellowish brown. Arista weak. Facial carina yellowish, developed, 1/2 length as face.

Thorax (Fig. 5D, F): Mesonotum and scutellum yellowish. Acrostichal setulae in ca 8 irregular rows. Halter yellowish brown. Wings light brown and appreciably hyaline.

Abdomen (Fig. 5D, F): Tergites yellowish.

Male terminalia (Fig. 12): Epandrium with ca 23 setae near posterior margin and ventral corner on each side (Fig. 12A, B). Surstylus broad, with ca 12 fine peg-like prensisetae on caudal margin, and numerous setae on outer and inner surface (Fig. 12A, B). Hypandrium pubescent near paramedian setae (Fig. 12C, D).

Measurements [holotype $\widehat{\partial}$ (range in $3 \widehat{\jmath})$, in $\mathrm{mm}$ ]: $\mathrm{BL}=2.36(2.33-2.44), \mathrm{Th}=$ 0.978 (1.02-1.07), $\mathrm{WL}=2.33(2.07-2.30)$, WW $=0.99(0.99-1.02)$.

Indices: $\mathrm{arb}=2 / 1(2 / 1)$, avd $=0.88(0.67-0.88)$, $\mathrm{adf}=0.97(0.97-0.99), \mathrm{flw}=$ $1.56(1.51-1.62), \mathrm{FW} / \mathrm{HW}=0.36(0.35-0.37), \mathrm{ch} / \mathrm{o}=0.12(0.11-0.13)$, prorb $=0.82$ $(0.95-0.98), \mathrm{rcorb}=0.50(0.50-0.52), \mathrm{vb}=0.73(0.47-0.52), \mathrm{dcl}=0.57(0.71-0.72)$, presctl $=0.36(0.42-0.44)$, sctl $=1.873(1.62-1.72)$, sterno $=0.43(0.41-0.43)$, or- 
bito $=0.48(0.48-1.07), \mathrm{dcp}=0.41(0.40-0.42)$, sctlp $=0.77(0.76-0.82), \mathrm{C}=3.79$ (3.76-4.09), $4 \mathrm{c}=0.59(0.53-0.60), 4 \mathrm{v}=1.69(1.72-1.80), 5 \mathrm{x}=0.86(0.86-0.95)$, $\mathrm{ac}=1.36(1.35-1.71), \mathrm{M}=0.31(0.29-0.32), \mathrm{C} 3 \mathrm{~F}=0.60(0.56-0.58)$.

Etymology. From the Latin word: "sinuatus", referring to the paramere, which is curved subbasally.

Distribution. China (Liaoning).

\section{Scaptodrosophila tanyrhina sp. nov.}

http://zoobank.org/20E556DF-CD47-4ACD-AB29-F9C8ADCA351B

Figs 6A, B, C, 13

Material examined. Holotype. CHInA: đิ (SCAU, No. 126401), Menglun, Mengla, Yunnan, $21^{\circ} 41^{\prime} \mathrm{N}, 101^{\circ} 25^{\prime} \mathrm{E}$, alt. 780 m, 17.iv.2007, HW Chen, JJ Gao. Paratypes. China: 7ð, 5 ㅇ (SCAU, Nos 126402-11, 110930-31), Menglun, Mengla, Yunnan, $21^{\circ} 41^{\prime} \mathrm{N}, 101^{\circ} 25^{\prime} \mathrm{E}$, alt. $780 \mathrm{~m}, 17 . i v .2007$, HW Chen, JJ Gao; 20, 1 ㅇ (SCAU,

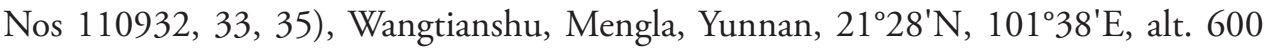
m, 23.iv.2007, HW Chen; $1 \delta^{\Uparrow}$ (SCAU, No. 110934), Guanglei, Mengla, Yunnan, $21^{\circ} 38^{\prime} \mathrm{N}, 101^{\circ} 10^{\prime} \mathrm{E}$, alt. $620 \mathrm{~m}, 21 . \mathrm{iv} .2016$, YQ Liu.

Diagnosis. Paramere broadened basally, finger-like in lateral view, with ca 6 sensillae subbasally and fine setae medially (Fig. 13C, D); gonopods undeveloped; aedeagus broadened apically, curved in lateral view (Fig. 13C, D).

Description. $(\hat{\partial},+)$ Head (Fig. 6A): Frons yellowish brown. Pedicel yellowish brown. First flagellomere yellowish. Facial carina yellowish, short, 1/3 length as face.

Thorax (Fig. 6B, C): Mesonotum and scutellum yellowish brown. Acrostichal setulae in ca 8 regular rows. Halter yellowish brown. Wings yellowish.

Abdomen (Fig. 6B, C): Tergite yellowish brown. Sternites yellowish.

Male terminalia: Epandrium with ca 13 setae near posterior margin and ventral corner on each side (Fig. 13A, B). Surstylus small with ca 9 peg-like prensisetae on caudal margin, and ca 6 setae on outer and inner surface (Fig. 13A, B). Hypandrium glabrous (Fig. 13C, D).

Female terminalia (Fig. 13E): Oviscapt with 3 long subapical trichoid ovisensilla, ca 19 peg-like ovisensilla on each side on ventral margins and 4 trichoid ovisensilla on dorsal.

Measurements [holotype $\widehat{\partial}$ (range in 5 $\hat{\sigma}, 59$ ), in $\mathrm{mm}$ ]: $\mathrm{BL}=1.87(1.80-2.07$, 1.80-2.13), ThL = $0.93(0.93-1.00,0.93-1.00), \mathrm{WL}=2.00(2.00-2.20,2.00-2.20)$, $\mathrm{WW}=0.80(0.80-0.87,0.73-0.80)$.

Indices: $\mathrm{arb}=2 / 1(2 / 1)$, avd $=0.75(0.60-1.00)$, adf $=2.00(1.50-2.50)$, flw $=$ $2.00(1.50-2.50), \mathrm{FW} / \mathrm{HW}=0.44(0.40-0.47), \mathrm{ch} / \mathrm{o}=0.10(0.09-0.10)$, prorb $=$ $1.00(0.67-1.00), \mathrm{rcorb}=0.40(0.33-0.50), \mathrm{vb}=0.30(0.25-0.50), \mathrm{dcl}=0.70(0.60$ $0.80)$, presctl $=0.60(0.40-0.64)$, sctl $=1.18(1.08-1.25)$, sterno $=0.60(0.50-0.78)$, orbito $=0.80(0.50-0.67), \mathrm{dcp}=0.27(0.30-0.36)$, sctlp $=0.67(0.67-1.00), \mathrm{C}=2.92$ $(2.71-3.27), 4 \mathrm{c}=1.00(0.85-1.18), 4 \mathrm{v}=2.33(2.15-2.58), 5 \mathrm{x}=1.60(1.33-1.80)$, $\mathrm{ac}=3.00(2.20-2.67), \mathrm{M}=0.67(0.57-0.73), \mathrm{C} 3 \mathrm{~F}=0.25(0.25-0.38)$. 

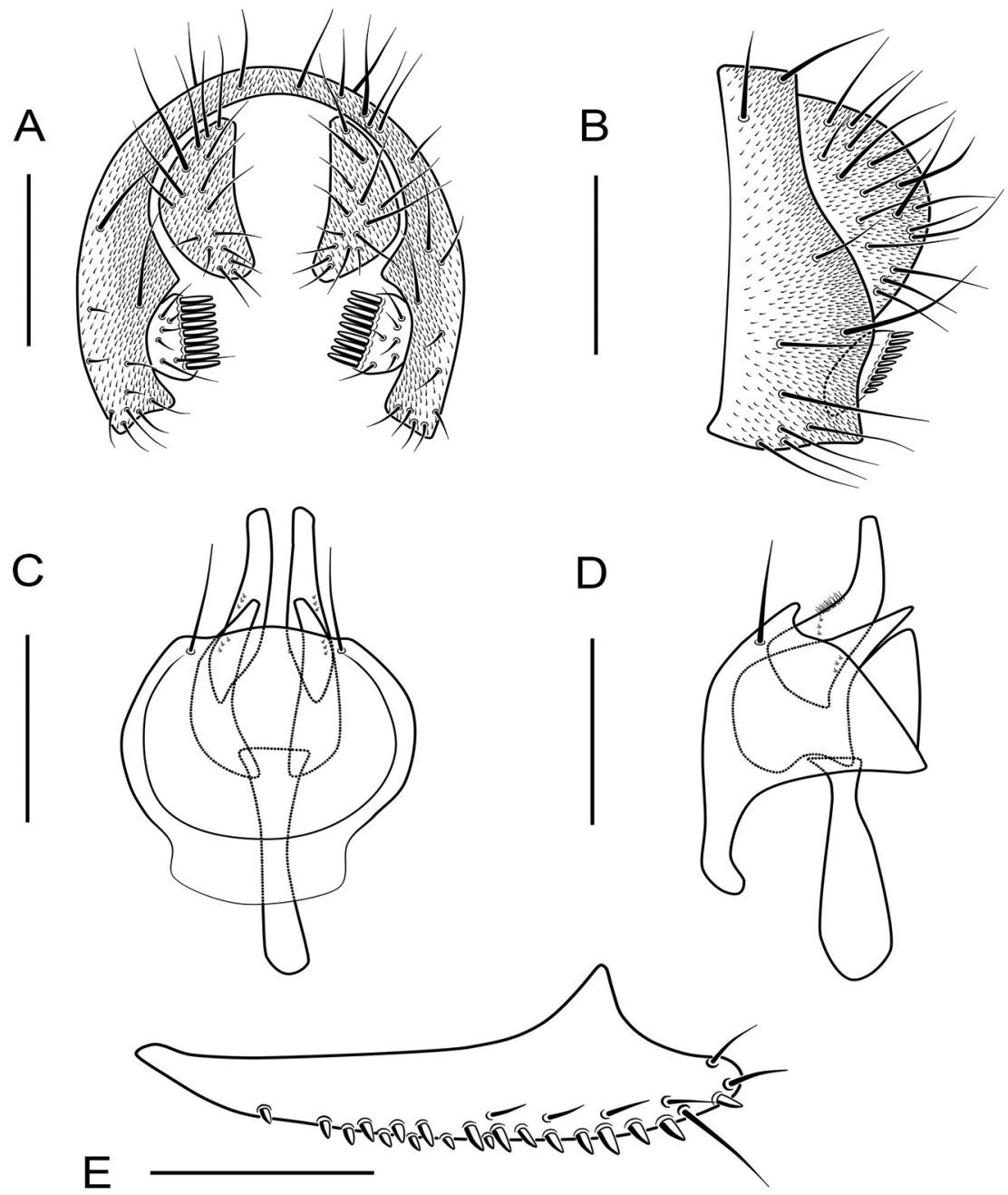

Figure 13. Scaptodrosophila tanyrhina sp. nov. O. A Epandrium, surstylus and cercus (posterior view) B epandrium, surstylus and cercus (lateral view) $\mathbf{C}$ hypandrium, aedeagus, aedeagal apodeme, paramere and gonopods (ventral view) D hypandrium, aedeagus, aedeagal apodeme, paramere and gonopods (lateral view) E oviscapt (lateral view). Scale bars: $0.1 \mathrm{~mm}$.

Etymology. A combination of the Latin words: "tanaos" + "rhinos", referring to the developed carina.

Distribution. China (Yunnan).

Key to examined species of the riverata group

\section{Adults (males)}

1 Frons reddish brown; clypeus reddish brown

S. puncticeps (Okada)

- $\quad$ Frons yellowish brown; clypeus yellowish brown 
2 Surstylus bifurcated (Fig. 7A, B) S. riverata (Singh \& Gupta)

- $\quad$ Surstylus not bifurcated 3

3 Dorsal 2 peg-like prensisetae of surstylus separated from each other (Fig. 9A, B) S. abdentata sp. nov.

- Surstylus peg-like prensisetae continuous ...................................................... 4

$4 \quad$ Gonopods with many finely acanthoid processes .....................................5

- Gonopods lacking finely acanthoid processes .............................................6

$5 \quad$ Third to sixth tegites brown (Fig. 5C) .................. s. serrateifoliacea sp. nov.

- $\quad$ Third to sixth tegites yellowish (Fig. 5D) ........................ S. sinuata sp. nov.

6 Paramere black...................................................... S. cederholmi (Okada)

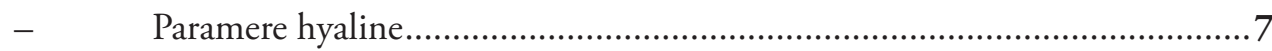

7 Paramere broadened distally in lateral view (Fig. 10C, D)

S. platyrhina sp. nov.

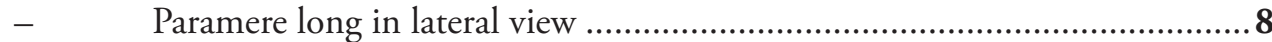

8 Paramere club-shaped ....................... S. paraclubata (Sundaran \& Gupta)

- $\quad$ Paramere finger-like in lateral view...........................................................9

9 Paramere with fine setae medially (Fig. 13C, D) ........ S. tanyrhina sp. nov.

- $\quad$ Paramere lacking fine setae.................................................. S. crocata (Bock)

\section{Acknowledgements}

We thank the reviewer Prof. PM O'Grady (Cornell University, USA) for the detailed corrections, and all the members of our laboratory (SCAU) for helping us in all the fieldwork. The parts of DNA sequences were provided by the Southern China DNA Barcoding Center, Kunming Institute of Zoology, China. This study was supported by the National Natural Science Foundation of China (Nos. $31672321,31093430)$.

\section{References}

Ashley HK, Bradley JS (2017) Manual of Afrotropical Diptera. Vol. 2. Nematocerous Diptera and lower Brachycera. Biodiversity for Life, South African National Biodiversity Institute. Pretoria, South Africa, 1361.

Bächli G (2019) The database on Taxonomy of Drosophilidae. http://taxodros.uzh.ch/ [last update: Jul 2019]

Bock IR (1976) Drosophilidae of Australia I. Drosophila (Insecta: Diptera). Australian Journal of Zoology 30: 46-94.

Bock IR (1984) A new species of the inornata group, genus Drosophila Fallén (Diptera: Drosophilidae). Journal of the Australian Entomological Society 23: 141-143. https://doi. org/10.1111/j.1440-6055.1984.tb01928.x 
Bock IR, Parsons PA (1978) The subgenus Scaptodrosophila (Diptera: Drosophilidae). Systematic Entomology 3: 91-102. https://doi.org/10.1111/j.1365-3113.1978.tb00106.x

Burla H (1954) Zur Kenntnis der Drosophiliden der Elfenbeinkuste (Französisch West-Afrika). Revue Suisse de Zoologie 61 (Supplement): 1-218. https://doi.org/10.5962/bhl. part.75413

Chen HW, Toda MJ (2001) A revision of the Asian and European species in the subgenus Amiota Loew (Diptera, Drosophilidae) and establishment of species-groups based on phylogenetic analysis. Journal of Natural History 35: 1571-1563. https://doi. org/10.1080/002229301317067665

DeSalle R, Egan MG, Siddall M (2005) The unholy trinity: taxonomy, speciesde limitation and DNA barcoding. Philosophical Transactions of the Royal Society of London, Series B 360: 1905-1916. https://doi.org/10.1098/rstb.2005.1722

Duda O (1923) Die orientalischen und australischen Drosophiliden-Arten (Dipteren) des ungarischen National-Museums zu Budapest. Annales Musei Nationalis Hungarici 20: 24-59.

Folmer O, Black MB, Hoeh W, Lutz RA, Vrijenhoek RC (1994) DNA primers for amplification of mitochondrial cytochrome $c$ oxidase subunit I from diverse metazoan invertebrates. Molecular Marine Biology and Biotechnology 3: 294-299.

Kumar S, Stecher G, Tamura K (2016) MEGA7: molecular evolutionary genetics analysis version 7.0 for bigger datasets. Molecular Biology and Evolution 33: 1870-1874. https://doi. org $/ 10.1093 / \mathrm{molbev} / \mathrm{msw} 054$

Liu, YQ, Chen, HW (2018) The genus scaptodrosophila duda part II: the coracina species group from east asia, with morphological and molecular evidence (diptera, drosophilidae). Zookeys 736: 119-148. https://doi.org/10.3897/zookeys.736.13682

Liu, YQ, Gao, QS, Chen, HW (2017) The genus scaptodrosophila duda part I: the brunnea species group from the oriental region, with morphological and molecular evidence (diptera, drosophilidae). ZooKeys 671: 87-118. https://doi.org/10.3897/zookeys.671.11275

Mather WB (1955) The genus Drosophila (Diptera) in eastern Queensland I. Taxonomy. Australian Journal of Zoology 3: 545-582. https://doi.org/10.1071/ZO9550545

McAlpine JF (1981) Morphology and terminology - adults. In: McAlpine JF, Peterson BV, Shewell GE, Teskey HJ, Vockeroth JR, Wood DM (Eds) Manual of Nearctic Diptera. Vol. 1. Monograph 27. Agriculture Canada Research Branch, Ottawa, 9-63.

Nei M, Kumar S (2000) Molecular evolution and phylogenetics. Oxford University Press, New York, 33 pp.

Okada T (1956) Systematic Study of Drosophilidae and allied families of Japan. Gihodo Co. Ltd., Tokyo, 1-183.

Okada T (1988) Family Drosophilidae (Diptera) from the Lund University Ceylon Expedition in 1962 and Borneo collections in 1978-1979. Entomologica Scandinavica Supplement 30: $134-135$.

Papp L, Rácz O, Bächli G (1999) Revision of the European species of the Scaptodrosophila rufifrons group (Diptera, Drosophilidae). Mitteilungen der Schweizerische Entomologischen Gesellschaft. Bulletin de la Société Entomologique Suisse 72: 105-117. 
Parsons PA, Bock IR (1978) Australian Endemic Drosophila III. The inornata species group. Australian Journal of Zoology 26: 83-90. https://doi.org/10.1071/ZO9780083

Sarkar IN, Thornton JW, Planet PJ, Figurski DH, Schierwater B, DeSalle R (2002) An automated phylogenetic key for classifying homeoboxes. Molecular Phylogenetics and Evolution 24: 388-399. https://doi.org/10.1016/S1055-7903(02)00259-2

Singh BK, Gupta JP (1977) The subgenus Drosophila (Scaptodrosophila) in India (Diptera: Drosophilidae). Oriental Insects 11: 239-241. https://doi.org/10.1080/00305316.1977 .10434540

Sundaran AK, Gupta JP (1991) A revision of the Indian species of subgenus Scaptodrosophila (Diptera: Drosophilidae). Zoologica Scripta 20: 291-299. https://doi. org/10.1111/j.1463-6409.1991.tb00293.x

Tamura K, Stecher G, Peterson D, Filipski A, Kumar S (2013) MEGA6: Molecular Evolutionary Genetics Analysis Version 6.0. Molecular Biology and Evolution 30: 2725-2729. https://doi.org/10.1093/molbev/mst197

Thompson JD, Higgins DG, Gibson TJ (1994) Clustal W: improving the sensitivity of progressive multiple sequence alignment through sequence weighting, position specific gap penalties and weight matrix choice. Nucleic Acids Research 22: 4673-4680. https://doi. org/10.1093/nar/22.22.4673

Throckmorton LH (1962) The Problem of Phylogeny in the Genus Drosophila. The University of Texas Publication 6205: 207-343.

Tsacas L, Chassagnard MT (1976) Identité de Drosophila brunnea de Meijere et description de nouvelles espèces orientales et africaines à pointe du scutellum blanche (Diptera, Drosophilidae). Bulletin Zoologisch Museum Universiteit van Amsterdam 5: 89-98.

Tsacas L, Chassagnard MT, David JR (1988) A new Afrotropical species-group of anthophilic Drosophila in the subgenus Scaptodrosophila (Diptera: Drosophilidae). Annales de la Société Entomologique de France 24: 181-202.

Wang BC, Park J, Watabe H, Gao JJ, Xiangyu JG, Aotsuka T, Chen HW, Zhang YP (2006) Molecular phylogeny of the Drosophilavirilis section (Diptera: Drosophilidae) based on mitochondrial and nuclear sequences. Molecular Phylogenetics and Evolution 40: 484500. https://doi.org/10.1016/j.ympev.2006.03.026

Wheeler MR (1949) XII. The subgenus Pholadoris (Drosophila) with descriptions of two new species. The University of Texas Publication 4920: 143-156.

Wheeler MR, Takada H (1966) The Nearctic and Neotropical Species of Scaptomyza Hardy (Diptera, Drosophilidae). The University of Texas Publication 6615: 37-78.

Zhang W X, Toda MJ (1992) A new species-subgroup of the Drosophila immigrans speciesgroup (Diptera, Drosophilidae), with description of two new species from China and revision of taxonomic terminology. Japanese Journal of Entomology 60: 839-850.

Zhao F, Gao JJ, Chen HW (2009) Taxonomy and molecular phylogeny of the Asian Paraleucophenga Hendel (Diptera: Drosophilidae). Zoological Journal of the Linnean Society 155: 616-629. https://doi.org/10.1111/j.1096-3642.2008.00450.x 